\title{
An adaptive scale Gaussian filter to explain White's illusion from the viewpoint of lightness assimilation for a large range of variation in spatial frequency of the grating and aspect ratio of the targets
}

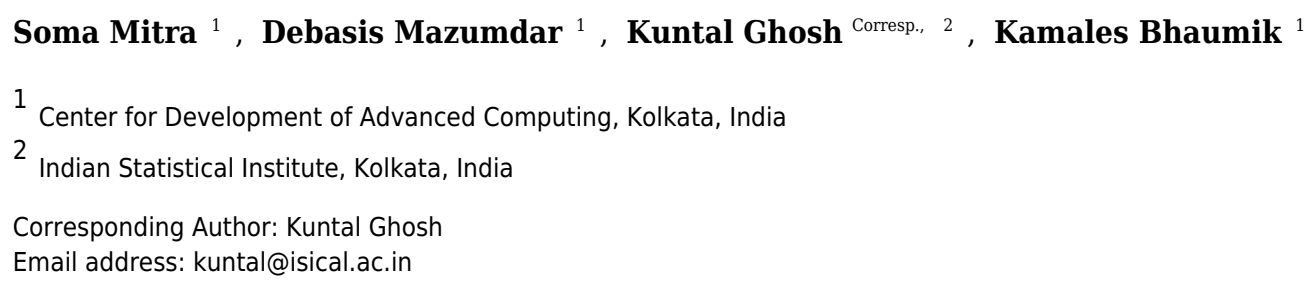

The variation between the actual and perceived lightness of a stimulus has strong dependency on its background, a phenomena commonly known as lightness induction in the literature of visual neuroscience and psychology. For instance, a gray patch may perceptually appear to be darker in a background while it looks brighter when the background is reversed. In the literature it is further reported that such variation can take place in two possible ways. In case of stimulus like the Simultaneous Brightness Contrast (SBC), the apparent lightness changes in the direction opposite to that of the background lightness, a phenomenon often referred to as lightness contrast, while in the others like neon colour spreading or checkerboard illusion it occurs opposite to that, and known as lightness assimilation. The White's illusion is a typical one which according to many, does not completely conform to any of these two processes. This paper presents the result of quantification of the perceptual strength of the White's illusion as a function of the width of the background square grating as well as the length of the gray patch. A linear filter model is further proposed to simulate the possible neurophysiological phenomena responsible for this particular visual experience. The model assumes that for the White's illusion, where the edges are strong and quite a few, i.e. the spectrum is rich in high frequency components, the inhibitory surround in the classical Difference-of-Gaussians (DoG) filter gets suppressed, and the filter essentially reduces to an adaptive scale Gaussian kernel that brings about lightness assimilation. The linear filter model with a Gaussian kernel is used to simulate the White's illusion phenomena with wide variation of spatial frequency of the background grating as well as the length of the gray patch. The appropriateness of the model is presented through simulation results, which are highly tuned to the present as well as earlier psychometric results. 
An adaptive scale Gaussian filter to explain White's illusion from the viewpoint of lightness assimilation for a large range of variation in spatial frequency of the grating and aspect
ratio of the targets

\author{
Soma Mitra ${ }^{1}$,Debasis Mazumdar ${ }^{1}$, Kuntal Ghosh $^{2}$ and Kamales Bhaumik ${ }^{1}$ \\ ${ }^{1}$ Center for Development of Advanced Computing, Kolkata, India \\ ${ }^{2}$ Indian Statistical Institute, Kolkata, India
}

\title{
8 Abstract
}

9 The variation between the actual and perceived lightness of a stimulus has strong dependency on \\ Introduction} its background, a phenomena commonly known as lightness induction in the literature of visual neuroscience and psychology. For instance, a gray patch may perceptually appear to be darker in a background while it looks brighter when the background is reversed. In the literature it is further reported that such variation can take place in two possible ways. In case of stimulus like the Simultaneous Brightness Contrast (SBC), the apparent lightness changes in the direction opposite to that of the background lightness, a phenomenon often referred to as lightness contrast, while in the others like neon colour spreading or checkerboard illusion it occurs opposite to that, and known as lightness assimilation. The White's illusion is a typical one which according to many, does not completely conform to any of these two processes. This paper presents the result of quantification of the perceptual strength of the White's illusion as a function of the width of the background square grating as well as the length of the gray patch. A linear filter model is further proposed to simulate the possible neurophysiological phenomena responsible for this particular visual experience. The model assumes that for the White's illusion, where the edges are strong and quite a few, i.e. the spectrum is rich in high frequency components, the inhibitory surround in the classical Difference-of-Gaussians (DoG) filter gets suppressed, and the filter essentially reduces to an adaptive scale Gaussian kernel that brings about lightness assimilation. The linear filter model with a Gaussian kernel is used to simulate the White's illusion phenomena with wide variation of spatial frequency of the background grating as well as the length of the gray patch. The appropriateness of the model is presented through simulation results, which are highly tuned to the present as well as earlier psychometric results.

Studies on visual illusions generally help in the formulation and testing of empirical models on visual perception. Plausible neural circuit, on the basis of direct or indirect evidences from 
neurophysiology, is often cited as a support to the empirical model. For some simple visual illusions, the visual presentations consist of a Background (uniform field across the display) and the Target Patches at specified colour values. A common example of such an illusion is the "Simultaneous Brightness Contrast", in which two targets with identical gray values are placed in different backgrounds. Target in the dark background appears lighter than the target in the white background. This is nicely explained with the empirical model of lateral inhibition (Kuffler, 1953). Here one assumes that the receptive field of, say a ganglionic cell, spread over the primary receptors in the retina, may be divided in a central and a peripheral region. For the same stimulus across the receptive field, the central and the peripheral regions send signals of opposite nature to the particular ganglion cell. If the former sends the excitatory signal, the later sends the inhibitory signal or vice versa. The model received wide attention because the results obtained from experimental neurophysiology seemed to provide a support to it (Ratliff and Hartline, 1959). ). The model was subsequently refined as "Difference of Gaussian" or DOG model (Rodieck and Stone, 1965) and also as "Laplacian of Gaussian" or LOG model (Marr, 1982). The lateral inhibitory process have already been used in developing model for various visual phenomena associated with lightness illusions (Macknik et al., 2000; Troncoso et al. 2005; Troncoso et. al., 2007; Troncoso et al. 2009)

Figure 1. Example of an illusion, exhibiting lightness assimilation. For details, see text.

There are a number of illusions for which the visual presentations contain another component, known as "Inducing Grating". Some of these, like the sinusoidal grating induction (McCourt, 1982), are explained with the help of lateral inhibition. However, there are others, like the square grating background in White's effect, which are not explainable with "lateral Inhibition" or DOG models. One such example is shown in figure 1, which has been adapted from de Weert and Spillmann (1995). Though this illusion was designed to show the asymmetry between induced lightness and induced darkness, it also beautifully illustrates the phenomenon of assimilation. Arrowhead 1 in the figure shows a target in the dark background, while arrowhead 2 shows a target (with same gray value as the previous one) in the white background. In this illusion the Target at arrow 1 appears darker than the Target at arrow 2, totally violating the principle of lateral inhibition. An alternative empirical model, known as "lightness assimilation", may be proposed in such cases. The term "lightness" needs to be defined. Appearance of an object depends not only on the luminance (luminous intensity over a given area and direction), but also on the reflectance of the object. Brightness is defined as "apparent luminance", while lightness is known as the "apparent reflectance". Brightness ranges from "dim" to "bright", while lightness ranges from "dark" to "light". So long the visible illumination is uniform, lightness follows brightness. The two properties are different when the scenario is non-uniformly illuminated. To explain the occurrence of illusions of the type, given in figure 1, one has to assume that the targets assimilate lightness from the surrounding or in other words the lightness at any point on the target patch is obtained by averaging the lightness over its surrounding. Such an averaging, also known as smoothing, is generally simulated through a Gaussian filter. Hence there is a popular belief that the visual presentations are perceived by the human brain after being modulated either through a DOG filter (contrast) or through a Gaussian filter (assimilation) or through an weighted sum of both (Young, 1987). It is also possible that in reality the human visual perception may not follow either of these alternatives. However, there is a relentless controversy (Kingdom, 2011) between these two processes and it will continue until the exact 
83

84

85

86

87

88

89

90

91

92

93

94

95

96

97

98

99

100

101

102

103

104

105

106

107

108

109

110

111

112

113

114

115

116

117

118

119

120

121

122

123

124

125

126

127

128

neural correlates of the visual perception are experimentally well established.

Figure 2. Example of White's illusion. For details, see text.

The present paper contains the report of our experimental and theoretical studies on the wellknown "White's illusion" (White, 1979, White, 1981), as shown in figure 2. Here the inducing grating consists of alternate black and white bars of equal width, characterized by its spatial frequency (which is generally expressed in cpd or cycles of repetitions per degree visual angle). Two target patches of equal length and identical gray values are taken. Both are placed coaxially, one along a white bar and the other along a dark bar. It is observed that the target which is mostly surrounded by black bars looks darker than the target which is mostly surrounded by the white bar. Since the observations cannot be explained through the model of "lateral inhibition", a model of "assimilation" is invoked for the explanation. The model of assimilation provides support for the perception of White's illusion over an wide range. Our experimental data show that the standard deviation (also known as the scale factor) of the Gaussian filter remains constant for a particular value of the spatial frequency of the inducing grating for an wide range of length of the test patch. The question is whether the model of assimilation is adequate for all ranges of length and width of the target patch. For example, if the length of the target becomes comparable to (or smaller than) its width, would it be possible to account for the magnitude of White's illusion with a single Gaussian filter? The present paper shows that the answer is affirmative, but, of course, with a price. It is observed that once the length of the patch is comparable to its width, the scale factor is to be adapted to a higher value. Hence we have used an adaptive scale Gaussian filter to fit the data on White's illusion from the viewpoint of lightness assimilation for a large range of variation in spatial frequency of the grating and aspect ratio of the targets.

The organization of the paper is as follows. In the beginning, we present the results of psychometric tests in our laboratory, following the methodology of two earlier papers (Shi et al., 2013; Troncoso et al., 2005). We performed two different experiments. In the first experiment, we measured the effect of the illusion for five different spatial frequencies of the inducing grating, while keeping the length of the target as fixed. In the second experiment, we had varied the length of the target to four different values at two fixed spatial frequencies. Purpose of the first experiment is to estimate the illusory component of perception for various spatial frequencies. Motivation of the second experiment comes from our emphasis in establishing assimilation as a dominant feature of White's illusion. The section, entitled Materials and Methods, gives the experimental set up in detail. The section on Results provides the data obtained from our experiments and comparison of those with existing data. Subsequent section on Proposed Model describes how we have tried to explain the results with an adaptive scale Gaussian filter model. In the section on Discussion, we provide justification in proposing a new model in spite of the fact that there are good models from reputed groups working in this field. Finally there is a section on Conclusion.

\section{Materials and Methods}

We report here two sets of data, obtained from our experiments. Two relevant parameters for our 
129 experiments are the spatial frequency of the bars and the length of the target patches. Spatial 130 frequency is given in the unit of cpd or cycles per degree of angle subtended at the eye. Lengths 131 and breadths of the patches may be given in pixels or in degree. In the following paragraph, we 132 shall give the algorithm for the conversion of bar width to cpd or pixels to degree. In the first set, 133 the spatial frequency was varied from $2.97 \mathrm{cpd}$ to $0.368 \mathrm{cpd}$, whereas the target length was kept constant at 70 pixels. In the second set the target length was varied from 16 pixels to 2 pixels while the spatial frequency was held constant at $1.47 \mathrm{cpd}$ and $0.738 \mathrm{cpd}$ respectively. In both the cases psychometric tests were conducted with six subjects including three adult males and three adult females. Four were naïve while two were chosen from among the authors. Each experimental session was of about 30 minutes' duration and 5 such sessions completed a full cycle of experiment. Written consents were obtained from all the subjects. The experiments have been approved by Scientific and Technical Advisory Committee (STAC), C-DAC.

\section{Variation of spatial frequency of the inducing grating}

The experimental arrangements were designed identical to that described in Shi et al. (2013) and Troncoso et al. (2005). To stabilize the subjects' heads, a chin rest was placed at a distance $d$ from a linearized video monitor (HP Compaq LE 2002X with resolution 1024 x 1024 pixels). The value of $d$ was chosen as $57 \mathrm{~cm}$., because it may be shown from simple trigonometry that at such a distance an image of width (or length) of $1 \mathrm{~cm}$. subtends a visual angle of approximately 1 degree. By measuring the pixel width in centimeter and by counting number of cycles per centimeter, one may convert those to cpd. For example, for bars of width 2 pixels, there are 5.5 cycles within a distance of 1.85 centimeters. Similarly for bars of width 32 pixels, there are 2.5 cycles within a distance of 13.6 centimeters. Accordingly widths of 2, 4, 8, 12, 16 and 32 pixels would correspond to $2.97,1.47,0.738,0.493,0.368$ and 0.184 cpd respectively. Subjects binocularly viewed the visual presentations, keeping their heads fixed on the chin rest. In these experiments, the subjects visually compare the brightness or lightness of two targets, namely the standard and the comparator. In our experiments, the standard was a target, containing a number of segments. Each segment could be distinguished from the other by its intensity of gray value. For example, our striped standard was divided into 11 segments of varying intensity. These values were kept fixed during the entire experiment, although the order of appearance of these 11 segments within the standard was scrambled pseudo-randomly. In our experiments, the comparators are the targets of identical gray values, taken on the black and white bars and hence these are the generators of the White's illusion, as shown clearly in figure 2. It is clear from figure 2, that the comparators that appear darker are bordered by more black than white, whereas the comparators that appear lighter are bordered by more white than black. Though the gray values of various segments of the standard are kept fixed (although scrambled) during one set of experiment, the comparators are changed from trial to trial, either by varying its width or length. Width of a comparator is always equal to the width of the black and white bars of the inducing grating. Hence the variation of the width of the comparator is synonymous to the variation of the spatial frequency of the grating. Subjects visually compare between the comparator and the standard on an uniform background with $50 \%$ relative luminance (equivalent to a gray value of 128). At the beginning of each trial, the subject was instructed to fix attention on a central red cross $\left(1^{\circ}\right.$ within a $3.5^{\circ}$ fixation window). After a lapse of 1 second, both comparator and standard appeared on the screen simultaneously. One of them was centered at $7^{\circ}$ to the left while the other centered at $7^{\circ}$ to the right of the central cross. Two-alternative forced-choice (2AFC) 
174 paradigm, introduced by Fechner (1889), was used in these lightness discrimination experiments.

175 If the comparator appeared to be lighter than the standard, subjects had to press key number

176 One, otherwise they had to press key number Two. Figure 3(a) shows the screen design. The

177 comparator was designed such that the absolute gray values of the black bars, white bars and the

178 comparators were 0, 256 and 128 respectively. Perceived lightness of the comparators were

179 strongly influenced by the lightness of the surrounding bars. Since the widths of the coaxial bars

180 modulate the perceived lightness of the comparators, we considered five different widths. Figure

181 3(b) shows three such stimulus presentations. For the smallest width $(2.97 \mathrm{cpd})$, eleven number

182 of bars (white + dark) could be accommodated within the stimulus, whereas for the largest width

183 (0.368 cpd), the number of bars had to be reduced to 5. This variation in the number of bars was

184 necessary to ensure that the region of comparison always be within $7^{\circ}$ around the central cross

185 mark. Two red vertical indicator lines were displayed $6^{\circ}$ from the top or from the bottom end of

186 both the standard and the comparator, in order to confine the attention of the subjects within the

187 specific region of interest. This is shown in Figure 3(b) for three different cases. Stimuli

188 (comparator and standard) appeared on the display for a duration of 3 seconds. Subjects need not

189 had to wait to give their judgments till the stimuli disappeared from the display. Red lines

190 indicate the region of interest in the comparator to be judged against the nearest segment of the

191 standard. The random choice of the selection of the region of interest ensured unbiased and

192 uniform probability distribution. Perceived difference of brightness between the comparator and

193 the standard depends on the actual difference of brightness between those and also the

194 psychophysical effect on the subject. If the actual difference in the brightness of the co-occurring

195 comparator and the standard is zero, the apparent perceived difference is then entirely due to the

196 psychophysical effect.

197 To keep the subjects unbiased, alert and attentive and also to avoid the fatigue during the 198 experiments, various parameters were randomly changed during the display. A number of criteria

(a) The standard had 11 segments with absolute gray values 13, 36, 59, 82, 105, 128, 150, 173, 196, 219 and 242. Equivalently the relative luminance of these segments were 5\%, $14 \%, 23 \%, 32 \%, 41 \%, 50 \%, 59 \%, 68 \%, 77 \%, 86 \%$ and $95 \%$ respectively. (Relative luminance of $100 \%$ corresponds to absolute gray value 255). Number of variations $=11$

(b) The subjects were exposed to a light appearing comparator (coaxial with black bar and flanked by white bars on both sides) in one half of the trials and a dark appearing comparator (coaxial with white bar) in the other half of the trials. Number of variations $=$ 2.

(c) The comparator appeared half the time on the left and half the time on the right of the standard during a complete session. Number of variations $=2$.

(d) The fixation marker was presented half the time on the top of the screen and half the time at the bottom of the screen randomly. Number of variations $=2$.

(e) In order to have equal probability of occurrence of each variation, total number of trials in a sub-session should be $11 \times 2 \times 2 \times 2=88$. Instead we have taken the number of trials in a sub-session as $88 \times 2=176$, so that probability of occurrence of each combination is 2 .

One session of experiment consists of five sub-sessions. In each of the sub-session the spatial frequency of the grating was kept fixed. The frequency was varied over the range 2.97, 1.47, $0.738,0.493$ and $0.368 \mathrm{cpd}$. The length of the comparator was kept fixed at 70 pixels or 12.075 
degrees. So the total number of experiments in a session is $176 \times 5=880$. Several such stimuli are shown in figure $3(b)$.

213

214

Figure 3: (a) Screen design of the psychophysical experiment, (b) Three different stimulus presentations of the lightness discrimination experiment.

\section{Variation of the length of the comparators}

215

216

217

218

219

220

221

222

223

224

225

226

227

228

229

230

231

232

233

234

235

236

237

238

239

240

241

242

243

244

245

246

247

248

In a second experiment, we have tested whether the features of White's illusion are compatible with the model of "lightness assimilation" for an wide range of the length of the comparators. Over here the spatial frequency of the inducing grating is kept fixed, while the length of the comparators are gradually changed. We have repeated this experiment for two different values of spatial frequencies, namely $1.47 \mathrm{cpd}$ and $0.738 \mathrm{cpd}$. For each of these frequencies, the lengths of the comparators were varied over four values, namely, 16, 8, 4 and 2 pixels (corresponding to $2.76,1.38,0.69$ and 0.345 degrees). The procedure of the experiments was identical to the previous one. The same six subjects (three males and three females) performed the experiments under identical conditions. Each sub-session, as before, consisted of 176 trials. So the total session required 176x $4=704$ trials. The subjects gave their judgments following $2 \mathrm{AFC}$ protocol. Written consents were obtained from all the subjects. When the length of the comparator is very small (like 4 or 2 pixels), some of the sub-sessions were repeated by keeping the red indicator in one set, while removing the red indicator in the repeated set. No perceivable difference in the outcome of the experiment in the two sets was observed, indicating that there was no problem in the visibility of the targets in presence of the indicator.

\section{Results}

Experiments, with $2 \mathrm{AFC}$ protocol, determine the subjective response thresholds of the performers of the experiments, which are essentially the comparator intensities required to produce a given level of performance. Performance of the subject improves as the actual difference in intensity of the comparator and the standard increases. Purpose of these experiments is to measure two main parameters.

(a) "point of subjective equality" (PSE) : The actual intensities of the comparator and the standard, when these appear to be same to the subject.

(b) Subjective ability to just discriminate between the intensities of the comparator and the standard. The former is known as "bias", while the later determines the "discrimination threshold".

\section{Variation with spatial frequency}

Psychometric curves, given in figure 4(a) are obtained by fitting the data with logistic functions using a maximum likelihood procedure. The function FitPsycheCurveLogit (used in MATLAB) is designed to fit a basic psychometric curve using a general linear model. The function uses glmfit to fit a binomial distribution with a logit link function. It is basically a cumulative 
249 Gaussian. The mean and variance of the Gaussian are assigned as the subject "bias" and 250 subjective "discrimination threshold". The function may take up to four input parameters,

251

252

253

254

255

256

257

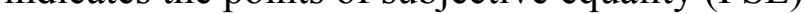

We have also fitted the same set of data with a modified function, developed by Wichmann and Hill (2001). They presented a cumulative Gaussian function with four parameters for fitting a psychometric function. These are mean, standard deviation, guess rate (g) and lapse rate (1). The parameters $g$ and 1 constrain the limits of the cumulative distribution that provides the sigmoid shape for the psychometric curve. The plot of the same set of average psychometric data is shown in figure 4(b). It is observed that the psychometric curves remain almost unaffected by this modification, though the family of curves appears to be more compact.

In figure 4(c) average Psychometric function for six experimenters has been plotted at spatial frequency $2.97 \mathrm{cpd}$. Observed deviations of perceived brightness for the experimenters are represented by the error bars.

In figure $4(d)$, the variation of the percentage illusory enhancement with spatial frequencies is plotted. The illusory enhancement or decrement is calculated from figure 4(b) at the point of subjective equality (PSE). For example from figure 4(b), the illusory enhancement at PSE for frequency of $0.368 \mathrm{cpd}$ is less than $5 \%$, whereas that for $2.97 \mathrm{cpd}$ is about $30 \%$. Illusory enhancement or decrement of lightness decreases as the width of the comparator is increased. The result is qualitatively similar to that obtained in Anstis (2005).

258

259

260

261

262

263

264

265

266

267

268

269

270

271

272

273

274

275

276
Figure 4. Psychophysical experimental result: Average Psychometric functions for different spatial frequencies are displayed in different colors. For a particular spatial frequency, the upper curve represents the condition when the comparator appears brighter and the lower curve represents the condition when the comparator appears darker. While drawing the Psychometric function, a pair of curves is placed symmetrically against the luminance difference value of 0 . Figure 4(a) gives the curves fitted with FitPsycheCurveLogit function. Figure 4(b) gives the curves fitted with modified function by Wichmann and Hill (2001). Figure 4(c) gives the average Psychometric function for six experimenters at a spatial frequency $2.97 \mathrm{cpd}$. The maximum and minimum deviation of perceived brightness for the individual experimenters are represented by the error bar at different luminance difference. Figure 4 (d) gives the Perceived enhancement in percentage of the points of subjective equality for different stimulus width.

Figure 5: Psychophysical experimental result: Average Psychometric functions for the different length of the gray patches are displayed in different colors. While drawing the Psychometric function, a pair of curves is placed symmetrically against the luminance difference value of 0 . In Figure 5(a) the width of the comparator is 8 pixels i.e. $0.738 \mathrm{cpd}$ and in Figure 5(b) the width of 


\section{Variation with aspect ratio of the comparators}

279

280

281

282

283

284

285

286

287

288

289

290

291

292

293

294

295

296

297

298

299

300

301

302

303

304

305

306

307

308

309

310

311

312

Psychometric curves, given in figure 5 are obtained by fitting the experimental data with the function FitPsycheCurveLogit. In the first set of experiment, as shown in figure 5(a), the comparator width was kept fixed at 8 pixels or $0.738 \mathrm{cpd}$, whereas the lengths of the comparators were varied over the set $(16,8,4,2)$ pixels or $(2.76,1.38, .69, .345)$ degrees. The second set of experiment is an exact repetition of the first one, only changing the width of the comparator at 4 pixels or $1.47 \mathrm{cpd}$. Results are shown in figure 5(b). One may calculate the illusory enhancement or decrement from the data on points of subjective equality (PSE) as depicted in the figures 5(a) and 5(b), following the same procedure that had been adopted in drawing the figure 4(d) from figure 4(b). It is clear from the figure 6 that for both the spatial frequencies, the percentage illusory enhancement or decrement of lightness is almost independent of the length of the comparator, though it varies with spatial frequency. If any set of four points are connected by piecewise straight lines, the net line becomes almost parallel to the abscissa. This property is exhibited by all the four lines in figure 6. Identical result was observed by Bakshi et al. (2016) over a much larger range of lengths. In figure 7 , lines, parallel to the abscissa, with an ordinate value equal to the average of the set of four points, are drawn. We assume a Null Hypothesis (H0): The percentage illusory enhancements (or decrements) for all lengths of the comparators at any specified spatial frequency are equal to the average value of the set. To check whether such $\mathrm{H} 0$ is tenable or not, we performed tests for goodness of fit for all four sets (each set containing four points) with $\chi^{2}$ distribution. The four $p$-values for the four sets are displayed in the figure 7. It is observed that all the $p$-values are greater than 0.99 , which leaves little scope to reject the Null Hypothesis. We, therefore, conclude: Percentage illusory enhancement (or decrement) in White's illusion depends on the spatial frequency of the inducing grating and is independent of the length of the comparator.

Figure 6. Perceived enhancement in percentage of the points of subjective equality for different target length.

Figure 7. Experimental data on \% illusory enhancement as a function of length of gray target in degrees has been plotted.

\section{Proposed Model}

To explain the observations on White's illusion, we propose a simple model of assimilation, which is equivalent to an averaging over the neighbourhood. To perform that averaging, we propose to filter the image data with a two-dimensional Gaussian $G(x, y)$ having only a single free parameter, namely, the standard deviation or scale factor $\sigma$ as given below 


$$
G(x, y)=\frac{1}{\sigma \sqrt{2 \pi}} e^{-\frac{\left(x^{2}+y^{2}\right)}{2 \sigma^{2}}}
$$

From the first set of experiments on White's illusion in which the spatial frequency was varied over five values, as depicted in figures 4(a) or 4(b), it is possible to estimate the illusory enhancements (decrements) at various frequencies as illustrated in figure 4(d). To check whether the simple Gaussian averaging model, as proposed above, is adequate to simulate the experimentally obtained values of illusory enhancement, as given in figure 4(d), one may proceed as follows. One may convolve the digital data of the image with the proposed Gaussian filter in order to measure the absolute gray value of the central point of the comparator after convolution. Since we know the actual value of the brightness/lightness of the comparator, we may easily evaluate the illusory enhancement (decrement) in percentage as depicted by our model. We call this value as the convolution response (\%). By adjusting the value of $\sigma$ we bring it as close as possible to the experimentally measured values of illusory enhancement (\%). Though a simple Gaussian averaging may reproduce the experimental values, the results for all the spatial frequencies, however, can not be simulated with a single value of $\sigma$. This is not surprising, because as the widths of the grating bars are increased, the corresponding mask sizes also increase. Consequently the scale factors $(\sigma)$ should also increase. To get good fit with experiment, the values of $\sigma$ were chosen as $(3.6,3.0,2.3,1.4$ and 0.8$)$ for spatial frequencies $(0.368,0.493,0.738,1.47$ and $2.97 \mathrm{cpd})$ respectively. The experimentally obtained illusory enhancement (or decrement) and the convolution response (\%) after fitting the value of $\sigma$ are compared in figure 8 . The length of the comparator was kept fixed in this experiment at 70 pixels or 12.07 degrees.

313

314

315

316

317

318

319

320

321

322

323

324

325

326

327

328

329

330

331

332

333

334

335
Coming to the second part of the experiment, namely the variation of White's illusion with the change of length of the comparator at a fixed value of spatial frequency, it may be noted that we concluded on the basis of our psychometric experiments at the end of Results : Percentage illusory enhancement (or decrement) in White's illusion depends on the spatial frequency of the inducing grating and is independent of the length of the comparator. Guided by this conclusion, a simulation experiment was conducted with five spatial frequencies $(2.97,1.47,0.738,0.493$ and $0.368 \mathrm{cpd}$ ), wherein for each frequency, the length of the comparator was varied over fourteen values $(70,60,50,40,32,24,16,14,12,10,8,6,4$ and 2 pixels, or in other words $12.075,10.35,8.625,6.9,5.52,4.14,2.76,2.415,2.07,1.725,1.38,1.035,0.69$ and 0.345 degrees respectively). For this extended study, we did not perform the psychometric experiments as each one of these experiments is quite time-consuming. Instead, we took recourse to the simulation experiment with the cue that the convolution response (\%) should remain largely the same over various lengths of the comparator at any specified value of the spatial frequency. We are confident that even if the simulation experiments are replaced by psychometric experiments, the final conclusion would remain the same.

Figure 8. Variation of the illusory enhancement (\%) or the convolution response (\%) with spatial frequency of the inducing grating. The value of $\sigma$ is varied from 3.6 to 0.8 as the spatial frequency is increased. The simulated data is normalized against the intensity value of 128 . The continuous curves represent the experimental results while the dotted curves are the outcome of 
the computer simulation.

337

338

339

340

341

342

343

344

345

346

347

348

349

350

351

352

353

354

355

356

357

358

359

360

361

362

363

364

365

366

367

368

369

370

371

372

373

374

375

376

377

378

379

380

381

The major constraint in the simulation was to adjust the scale factor $\sigma$ such that the convolution response (\%) remains constant over all lengths of the comparator. Starting point of the simulation is to assume that the magnitude of the illusory enhancement (\%) for a comparator of length 70 pixels (obtained through our first experiment and shown in figure 4(d)) for any particular frequency should not vary due to the variation of the length of the comparator. The starting value of the scale factor is also taken from the fitted curve as given in figure 8 . The value of the spatial frequencies, corresponding length of the gray patch and the scale factor of the Gaussian kernels are presented in Table 1 . The mask sizes were chosen approximately 3 times the corresponding widths of the grating bars. The simulation keeps the convolution response (\%) constant over various values of the length of comparator by adapting to a suitable value of the scale of the Gaussian, as given in Table I. This is illustrated in figure 9. It may be noted that the fitted scale factor of the Gaussian kernel remains constant over a fairly large range of the comparator length. It starts increasing only when the length and breadth of the comparator are comparable to one another.

Though the data obtained from our simulation experiment are given in detail in the Table 1, it may give rise to a possible misunderstanding. It may appear, as if, the convolution had been performed with a Gaussian filter only on the comparators without taking into consideration the effect of such a convolution over the entire visual presentation. To avoid any such misunderstanding, a pictorial

Figure 9. Percentage convolution response for different length of the target while keeping the spatial frequency as fixed. In the graph, the red, blue, green, cyan and magenta curves show the simulated output at $2.97 \mathrm{cpd}, 1.47 \mathrm{cpd}, 0.738 \mathrm{cpd}, 0.493 \mathrm{cpd}$ and $0.368 \mathrm{cpd}$ respectively.

\section{Table 1. Parameters of the stimuli and the filter used in the simulation with varying spatial frequencies and the length of the gray patch}

presentation of the effect of Gaussian filter on standard, comparator and the inducing grating is included here. In figure 10(a), an example of White's illusion at four different frequencies is given. Illusion at one frequency is separated from the other by an uniform gray background. A line $\mathrm{AA}^{\prime}$

Figure 10. (a) White's illusion at four different frequencies separated from one another by a uniform gray background. 10 (b) and 10 (c) represent one dimensional intensity profile corresponding to the lines $\mathrm{AA}^{\prime}$ and $\mathrm{BB}^{\prime}$ respectively.

Figure 11. Shows the effect of Gaussian filtering with adaptive scale factors on figures 10(a), 10 (b) and 10 (c). The illusory effect at different frequencies can be differentiated by the naked eye. (a) gives convolved image of figure 10(a) with Gaussian filtering. Figures (b) and (c) shows 
382

383

384

385

386

387

388

389

390

391

392

393

394

395

396

397

398

399

400

401

402

403

404

405

406

407

408

409

410

411

412

413

414

415

416

417

418

419

420

421

422

423

424

425

426

427

the effect of Gaussian convolution on figures 10 (b) and 10 (c) respectively.

has been drawn parallel to the abscissa, such that the grating bars, coaxial with the comparators, are all white. Similarly another line BB' has been drawn such that the grating bars, coaxial with the comparators are all black. One-dimensional intensity profiles, corresponding to the lines AA' and $\mathrm{BB}^{\prime}$, are shown in figures 10 (b) and 10(c) respectively. In both of these figures the ordinate gives the absolute gray value of the image in a scale from 0 to 255 . There are four bunches of values corresponding to the four illusions considered in figure 10(a). The absolute gray value of the uniform background that had been introduced to separate one illusion from the other is not shown in the picture. Abscissa has been labeled as "distance in pixels". This is just to provide an information about the spatial frequencies of the gratings. Distance from one bunch to the other carries no physical relevance. Figures 11 (a), (b) and (c) show the effect of Gaussian filtering with adaptive scale factor on figure 10 (a), (b) and (c) respectively. The convolved image of figure 10(a), as given in figure 11(a), shows blurring of both comparators and the inducing grating. However, the illusory effect on the comparators at different frequencies can be differentiated by a naked eye. Blurring effect on the inducing grating reduces as the spatial frequency is increased. The blurring effect, as shown here, is also present in the convolution output of the ODOG model by Blakeslee and McCourt (1999;2004). The actual intensity of the comparators in absolute gray value is 128 . The figures 11(b)and 11(c) clearly show how the illusory enhancement through Gaussian filtering can be estimated from the diagram. For example, at a spatial frequency of $2.97 \mathrm{cpd}$, convolution output, as given in figures 11(b) and $11(\mathrm{c})$, shows that the illusory enhancement in absolute gray value is $(150.8-128)$ or 22.8 , whereas the illusory decrement is $(128-105)$ or 23 . Another important feature that comes out of these figures is that the magnitude of illusory enhancement or decrement decreases as the spatial frequency of the grating is decreased.

\section{Discussion}

Regarding our proposed model, it should be noted that there is no novelty or surprise in it. It is known for a long time that a large number of cases of visual illusion may be explained fully or partially by invoking assimilation, which has a close similarity to an averaging through Gaussian kernel. We have simply emphasized here that such an extremely simple model may lead to the explanation of White's illusion over a large variation in spatial frequency and length of the comparator. We have varied the length of the comparator from 70 pixels to 2 pixels to show how this simple model of averaging may reproduce the observations on White's illusion over an extensive range, while the popular model involving ODOG (Blakeslee and McCourt, 1999; 2004) fails near the upper limit of this range of length (Bakshi et al., 2016). Moreover, the present model involves a single free parameter, namely scale factor or $\sigma$. Fitting such a wide range of data by adjusting a single parameter is undoubtedly an example of a good engineering model. To establish this model as a paradigm in visual perception, we are to enquire further whether it is also supported by experimental neurophysiology of the visual system. It may be noted that the model involves three important features. (a) For assimilation we have used a simple Gaussian blurring. (b) The fitted scale factor varies over an wide range, indicating that in some situations information is collected and averaged over very large area. Such a long range 
428 interaction was not called for in the classical models. (c) The well-known antagonism between 429 the central and peripheral regions in the receptive field of the neurons is given up in the present 430 model in favour of a single Gaussian. In the following we are going to cite evidences from 431 neurophysiology for all these three features of our model.

432 (a) Assimilation is a Gaussian averaging: Gaussian averaging is omnipresent in every layer of 433 information collection in the retinal neuronal network. In fact in any connected network, through which the information is passed from one end of it to the other, stimulus at a point is spread through the entire network in a manner having close similarity with Gaussian averaging. A good example may be cited from the properties of resistive network in which a voltage input at any point is spread like Gaussian blurring. In the neural network of the retina itself, there are evidences of Gaussian averaging through bipolar cells, through horizontal cells and through amacrine cells.

440

441

442

(b) Variation of the scale factor over a large range: Studies on contrast started much earlier than the studies on assimilation. Experimental data and theoretical models on contrast indicate the existence of two different Gaussian averaging, one for the central and the other for the peripheral region of the receptive field. Experiments supported the belief that contrast is manifested as a local property of the network, which means the averaging are done over small areas. Consequently the value of $\sigma$ should be small. In our proposed model, we find that the fitted values of $\sigma$ vary widely, ranging from 0.8 to 13 . Classical works suggest that the averaging is done over a center-surround radius of less than 300 micrometers. As a basis to our model, the experimental data from neurophysiology is necessary to support the claim of averaging over a distance, much larger than 300 micrometers. Such data are pouring over in the recent years. Data from several laboratories indicate that (Yeonan-Kim and Bertalmio, 2016) there are some varieties of amacrine cells which collect information over a radius of more than 500 micrometers. Recent works on wiry amacrine cell reveal that (Manookin et al., 2015) soma of some of these cells collects information from a distance, which may be larger than 1000 micrometers or $1 \mathrm{~mm}$. Diameter of such a receptive field may exceed $2 \mathrm{~mm}$, which is larger than the classical estimate at least by an order of magnitude. These wide-field cells are also found to lack the property of center-surround antagonism. Receptive fields for these wiry amacrine cells were mapped and they were at least 10 times larger than the receptive fields of parasol ganglion cells. So the wide range of fitted value of $\sigma$ should not be considered as a mere fanciful hypothetical extrapolation. On the contrary the range corroborates to the recent data on neurophysiology.

Even if the existence of such wide field cells are accepted, one may wonder whether depending on the nature of the stimulus, different types of cells are invoked for averaging in different types of scenario. Alternatively, is it true that the same cell, depending on the visual presentation, goes on changing its value of $\sigma$ ? The experimental neurophysiology of the visual pathway is still in its infancy to provide a clear answer to these queries. We shall, however, revert back to this point little later.

(c) Well-known antagonism between central and peripheral region vanishes in the proposed model. What is the neural correlate/ of that phenomenon? Contrast sensitivity is so ubiquitous in sensory physiology, that it was predicted much before any advent in electrophysiology of neurons. Later in the very initial stage of experimental neurophysiology, the principle of lateral inhibition (existence of DOG filter) in explaining contrast sensitivity was so firmly established in visual perception that now-a-days it is accepted without any controversy. Examples of assimilation started gathering much later. Though the model of lateral inhibition is at least one 
step more complicated than the model of assimilation, acceptance of the latter is not spontaneous. Even today, DOG is a more natural choice than the averaging through a single Gaussian. Some people believe that the existence of DOG filter is supported by direct neurophysiological evidences. That belief, however, is not so well founded. Experiments vindicating the process of DOG are all based on the stimulus response relationship of the neurons in the visual pathway. For example, an electrode is placed inside, say, a ganglion cell. A strong spot of light is shown in the centre of its receptive field to elicit vigorous response in the ganglion cell. The response becomes milder as the radius of the spot of light is increased, showing the average response from the central region is antagonistic to the average response from the peripheral region. However, nobody has been able to prove that the neurons of peripheral region release inhibitory neurotransmitter to its presynaptic terminal. Two neurons communicate either through an electrical synapse or a chemical synapse or through some ephaptic coupling. In case of retinal lateral inhibition, no signature of inhibitory neurotransmission has ever been detected. We quote from Kramer and Davenport (2015) : "Despite decades of research, the feedback signal from horizontal cells to photoreceptors that generates lateral inhibition remains uncertain. GABA, protons, or an ephaptic mechanism have all been suggested as the primary mediator of feedback. However, the complexity of the reciprocal cone to horizontal cell synapse has left the identity of the feedback signal an unsolved mystery." On the other hand from a different set of experimental results, Shapley et al.(1990) concluded that "responsiveness of the visual system to contrast is not a result of center-surround interaction, or, in other words, of lateral inhibition (as in the standard textbook accounts........). Rather, the key to understanding dependence of response on contrast is to realize that contrast dependence is primarily a result of the automatic gain control that produces light adaptation."

In the light of the above discussion, we are trying to understand the phenomenon of visual perception with a fresh outlook, instead of getting biased with any existing model. Only experimental observation in the retinal network that can never be refuted is the Gaussian averaging. Averaging in the retinal network operates at different scales. It is done within a very short distance (central region, probably through bipolar cells), medium distance (peripheral region, probably through horizontal cells) or large distance (as mentioned in this paper, probably through amacrine cells). This is the first stage for information collection. In the next stage a primal sketch (Marr, 1982) of the visual scenario is constructed by following some rules of combination of these averages. The averages may combine positively or antagonistically, linearly or non-linearly following a single principle, namely the principle of survival of the individual in the process of evolution. Obviously the rules of combination should depend on the nature of the stimulus. In some situation central and peripheral averages may combine antagonistically. In some situation the antagonism may vanish and/or may be superseded by another average from a much larger area (known as extra classical receptive field). The locus of coding these rules is still elusive. It may be at a higher cortical level or it may even be controlled by the continuous feedback and feed forward exchanges between the primary receptors and a neuron at a higher level in the visual pathway, say at lateral geniculate nucleus. To get a noncontroversial neural correlates for the models of visual perception, we shall have to wait till experimental neurophysiology provides answer to these questions. Till then, we shall have to go through the process of careful data collection and prediction of empirical models. Reverting back to the discussion that we had postponed at the end of point (b) above, it appears that may be both of the alternatives are true. Existence of specialised cells which may sum up information from a long distance are indeed present, but not always called for. On the other hand the same neuron 
may dynamically adjust its scale factor for the survival of the organism. No fixed rules are followed while drawing even the primal sketch from the raw data. Visual perception is complicated by the fact that the raw visual information dictates the algorithm through which it should be processed for final computation.

Finally, we would like to discuss another tempting alternative model for visual perception, namely deconstruction of the visible image into its Fourier components by neurons in the circuit of visual pathway and later reconstructing the image at the cortical level from the amplitudes of the components and their phases (Campbell and Robson, 1968). The first and foremost requirement of such a model is to prove the existence of neurons which are finely tuned to various spatial frequencies. Such cells were discovered long back in the primary visual cortex of the cat and macaque monkeys (Maffei \& Fiorentini, 1973; DeValois, Albrecht, \& Thorell, 1982). So it is expected that the Fourier amplitudes of the visual image may be well represented among the cells in the visual cortex. It was also found that simple and complex cells of cat encode the phase information of the image (Spitzer and Hochstein, 1985). If both amplitude and phase information are retractable in the cortex, reconstruction of the original visual scenario should not be problematic.

We have digressed to this topic of Fourier decomposition, because it carries some relevance to the present topic. In a previous work (Mazumdar et al., 2016) we had studied the variation of the width of Mach bands with the nature of discontinuity in the intensity. We defined a term "sharpness of discontinuity" (SOD) and observed that an empirical DOG model, in which the scale factor of the surround Gaussian is a function of SOD, is able to reproduce the results. It was further shown in that paper that if the visual system is indeed capable of performing Fourier analysis, then SOD can be estimated. The model showed that the effect of surround suppression had to be reduced as the contrast at the edge increased. In the extreme limit of binary edges, where the contrast is maximum and represented by a step edge, no lateral inhibition takes place. Thus at high frequency, DOG gets converted into a Gaussian kernel leading to the vanishing of Mach band. Taking the cue from this previous work, we have applied that in this paper too. Since White's illusion involves many high frequency features, we conjectured that a model with total surround suppression with an adaptive scale factor may be appropriate for analysing the effect. It is, of course, merely a conjecture that has resulted into a good working model. There is no direct experimental observation supporting this conjecture.

\section{Conclusion}

For the bipedal apes, walking upright, the visual system is no doubt an extremely important sensor to gather information of the outside world. From any visual scenario, the organism for its survival has to identify its predators and preys. Depending on its survival strategy, the same scenario may be analysed for its details or may be grossly averaged out. Accordingly the final computation of the same scenario may differ widely from one another. Human beings, evolving over millions of years, have learned that the information from the same scenario may be computed through varieties of algorithms. However, though the computational algorithms may differ from one type of analysis to the other, probably the basic neuronal circuits in collecting the raw visual information do not change. Information collected at different layers are manipulated via different rules depending on the purpose of the analysis.

Proceeding with that belief, we feel that there is no necessity of having different neuronal circuits for visualising contrast or assimilation. Depending on the scenario, the same set of data may give rise to the perception of contrast or of assimilation. Such an idea was floated long back by 
Helson (1963), while working with a set of white bars on a gray background. He observed that by changing the spatial frequency of the bars, the perception moves from contrast to assimilation. Similar effect was observed by replacing the white bars with black bars. Another famous experiment by Reid and Shapley (1988) with disk and ring stimuli showed that decreasing the size of the surrounding area decreased contrast and increased assimilation. However, these authors were so obsessed by the limited size of the center-surround receptive field that they had to assume the process of assimilation as a post-retinal, perhaps cortical, phenomenon. With the recent findings on wide field amacrine cells, that obsession is removed. Thus we agree with Yeonan-Kim and Bertalmio $(2015,2016)$ that the existence of such wide field interneurons establishes that contrast sensitivity and lightness assimilation share the same neural locus.

467

468

469

470

471

472

473

474

475

476

477

478

479

480

481

482

483

484

485

486

487

488

489

490

491

\section{Additional Information and Declarations}

\section{Ethics}

The authors have followed the COPE guidelines for ethical responsibilities and written consent was obtained from all subjects.

\section{Acknowledgement}

The first two authors are thankful to the MeitY, Govt. of India for sponsoring the project titled 'Development of Human perception inspired algorithms for solving deeper problem in Image Processing and Computer vision ', Administrative approval No: DIT/R\&D/C-DAC/2(7)/2010 dated March 28, 2011. The authors would like to thank Ms. Gargi Bag, CDAC, Kolkata for helping in the development of the MATLAB code. The authors are also grateful to all the Reviewers for their valuable comments that helped in improving the work.

\section{References}

Anstis SM. 2005. White's effect in color, luminance and motion. In: Harris L \& Jenkin M (Eds): Seeing Spatial Form, Oxford University Press.

Bakshi, A, Roy, S, Mallick, A, Ghosh, K. 2016. Limitations of the Oriented Difference of Gaussian Filter in Special Cases of Brightness Perception Illusions. Perception 45: 328-36

Blakeslee B, McCourt ME. 1999. A multiscale spatial filtering account of the White effect, simultaneous brightness contrast and grating induction. Vision Res 39: $4361-4377$

Blakeslee B, McCourt ME. 2004. A unified theory of brightness contrast and assimilation incorporating oriented multiscale spatial filtering and contrast normalization. Vision Res 44: $2483-2503$ 
492

493

494

495

496

497

498

499

500

501

502

503

504

505

506

507

508

509

510

511

512

513

514

515

516

517

518

519

520

521

522

523

524

525

526

527

Campbell FW, Robson JG. 1968. Application of fourier analysis to the visibility of gratings. $J$ Physiol 197: 551-566

De Valois RL, Albrecht DG, Thorell LG. 1982. Spatial frequency selectivity of cells in macaque visual cortex. Vision Research 22: 545-559.

De Weert ChMM, Spillmann L. 1995. Assimilation: asymmetry between brightness and darkness. Vision Research 35: 1413-1420.

Fechner, Gustav Theodor.1889. Elemente der Psychophysik (2 Volumes) (2nd ed.). Leipzig: Breitkopf\&Härtel. Vol 2.

Helson H. 1963. Studies of anomalous contrast and assimilation. Journal of the Optical Society of America, 53: 179-184. http://dx.doi.org/10.1364/JOSA.

Kingdom F. 2011. Lightness, brightness and transparency: A quarter century of new ideas, captivating demonstrations and unrelenting controversy. Vision Res 51: 652-673

Kramer R H, Davenport C M. 2015. Lateral Inhibition in the Vertebrate Retina: The Case of the Missing Neurotransmitter. PLoS Biol 13(12): e1002322. doi:10.1371/journal.pbio.1002322;

Kuffler S W. 1953. Discharge patterns and functional organization of mammalian retina. $J$ Neurophysiol 16: 37-68

McCourt M E. 1982. A spatial frequency dependent grating-induction effect. Vision Research 22: $119-134$

Macknik S L, Martinez-Conde S, Haglund M M. 2000. The role of spatiotemporal edges in visibility and visual masking', Proceedings of the National Academy of Sciences of the United States of America 97:7556-7560

Maffei L, Fiorentini A. 1973. The visual cortex as a spatial frequency analyser. Vision Research 13: $1255-1267$

Manookin M B, Puller C, Rieke F, Neitz J, Neitz M. 2015. Distinctive receptive field and physiological properties of a wide-field amacrine cell in the macaque monkey retina, $J$. Neurophysiol 114: 1606-1616

Marr D. 1982.Vision: A Computational Investigation into the Human Representation and Processing of Visual Information. WH Freeman, New York

Mazumdar D, Mitra S, Ghosh K, Bhaumik K. 2016.A DOG filter model of the occurrence of Mach bands on spatial contrast discontinuities. Biological Cybernetics 110: 229-236

Ratliff, F., Hartline, H.K. 1959. The responses of Limulus optic nerve fibers to patterns of illumination on the receptor mosaic. Journal of General Physiology 42: 1241-1255 
528

529

530

531

532

533

534

535

536

537

538

539

540

541

542

543

544

545

546

547

548

549

550

551

552

553

554

555

556

557

558

559

560

561

Reid RC Jr1, Shapley R. 1988. Brightness induction by local contrast and the spatial dependence of assimilation. Vision Research 28: 115-132

Rodieck RW, Stone J. 1965.Analysis of receptive fields of cat retinal ganglion cells. $J$ Neurophysiol28: 833-849

Shapley R, Caelli T, Grossberg S, Morgan M, Rentschler I. 1990. "Computational theories of visual perception" in Visual Perception: The Neurophysiological foundations, ed. by Spillmann L and Werner J S, pp 417-448, Academic Press, Inc.California, USA.

Shi V, Cui J, Troncoso XG, Macknik SL, Martinez-Conde S. 2013.Effect of stimulus width on simultaneous contrast.PeerJ 1:e146 DOI 10.7717/peerj.146

Spitzer H, Hochstein S. 1985. Simple- and complex-cell response dependences on stimulation parameters. J Neurophsiol. https://doi.org/10.1152/jn.1985.53.5.1244

Troncoso XG, Macknik SL, Martinez-Conde S. 2005. Novel visual illusions related to Vasarely's "nested squares" show that corner salience varies with corner angle. Perception34:409-420

Troncoso X G, Tse P U, Macknik S L, Caplovitz G P, Hsieh P-J, Schlegel A A, OteroMillan J, Martinez-Conde S. 2007, 'BOLD activation varies parametrically with corner angle throughout human retinotopic cortex', Perception 36:808-820 DOI 10.1068/p5610

Troncoso X G, Macknik S L, Martinez-Conde S 2009. 'Corner salience varies linearly with corner angle during flicker-augmented contrast: a general principle of corner perception based on Vasarely's artworks', Spatial Vision 22:211-224 DOI 10.1163/156856809788313129

White M.1979. A new effect of pattern on perceived lightness. Perception, 8:413-416

White M. 1981. The effect of the nature of the surround on the perceived lightness of gray bars within square wave test gratings.Perception, 10:215-230

Wichmann FA, Hill NJ. 2001. The psychometric function: I. Fitting, sampling, and goodness of fit. Perception \& Psychophysics 63:1293-1313

Yeonan-Kim J, Bertalmio M. 2016. Retinal Lateral Inhibition Provides the Biological Basis of Long-Range Spatial Induction, PLoS ONE 11(12): e0168963. doi:10.1371/journal. Pone. 0168963

Yeonan-Kim J, Bertalmio M. 2015. Surrounding size effect on Brightness Contrast-toAssimilation ispredicted in retina, submitted to Journal of Vision. May be obtained from http://ip4ec.upf.edu/KimJOV15

Young RA.1987.The Gaussian Derivative model for spatial vision: I. Retinal mechanisms. 
562 Spatial Vision 2:273-293

563

564

565

566 


\section{Figure 1}

Example of an illusion, exhibiting lightness assimilation.

Figure 1. Example of an illusion, exhibiting lightness assimilation. For details, see text.
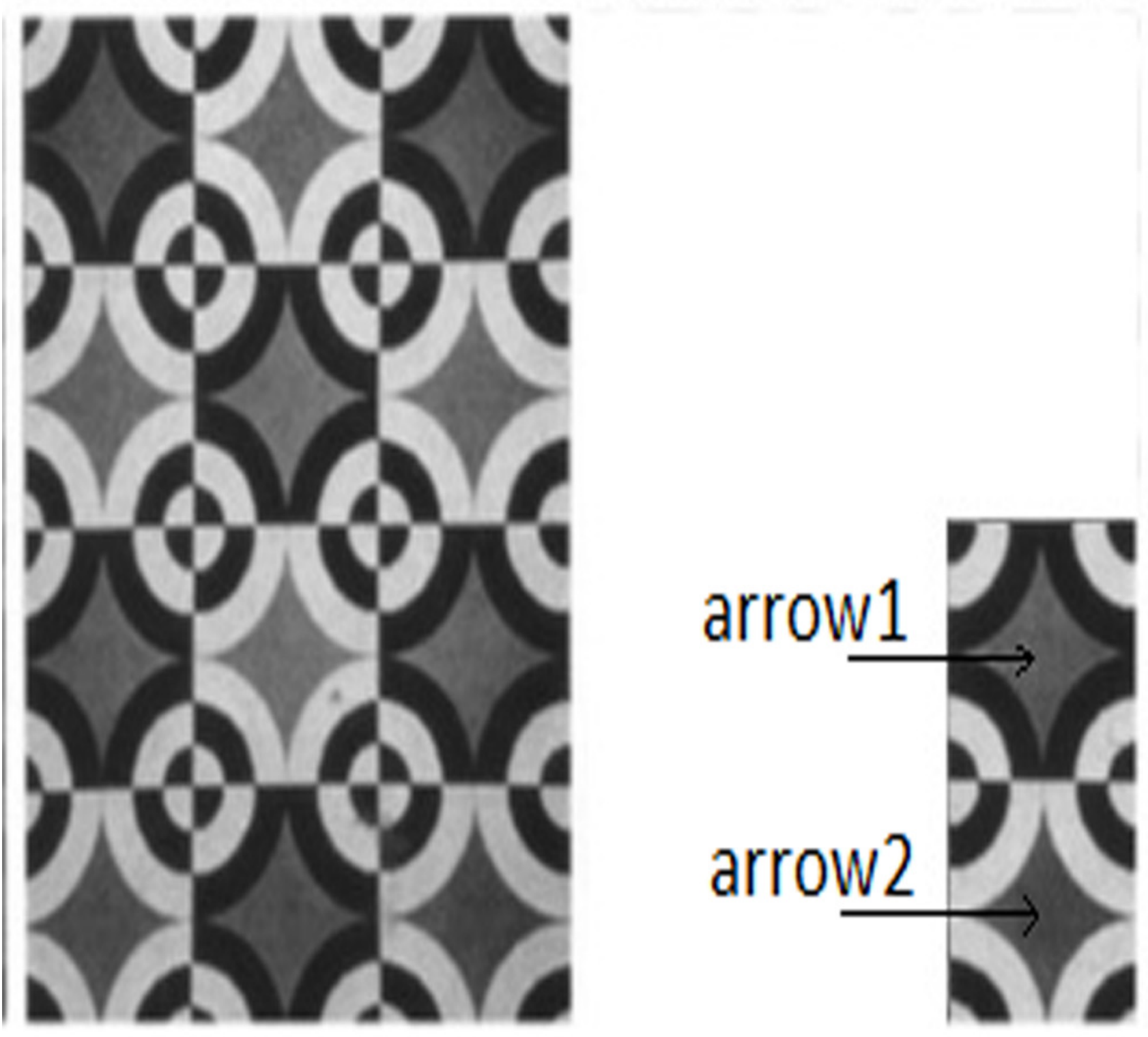


\section{Figure 2}

Example of White's illusion.

Figure 2. Example of White's illusion. For details, see text.

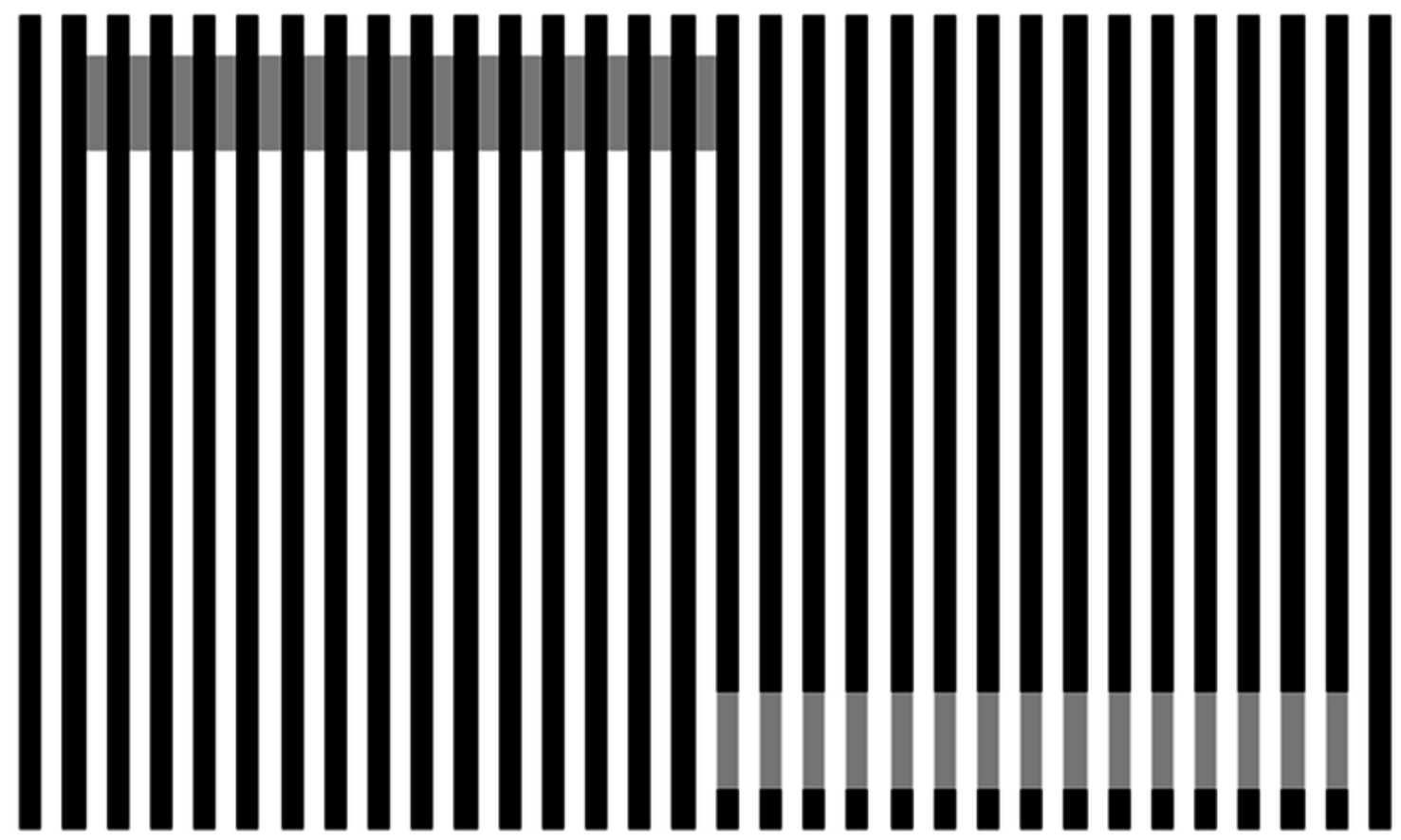




\section{Figure 3}

Screen design of the psychophysical experiment

Figure 3 : (a) Screen design of the psychophysical experiment, (b) Three different stimulus presentations of the lightness discrimination experiment.
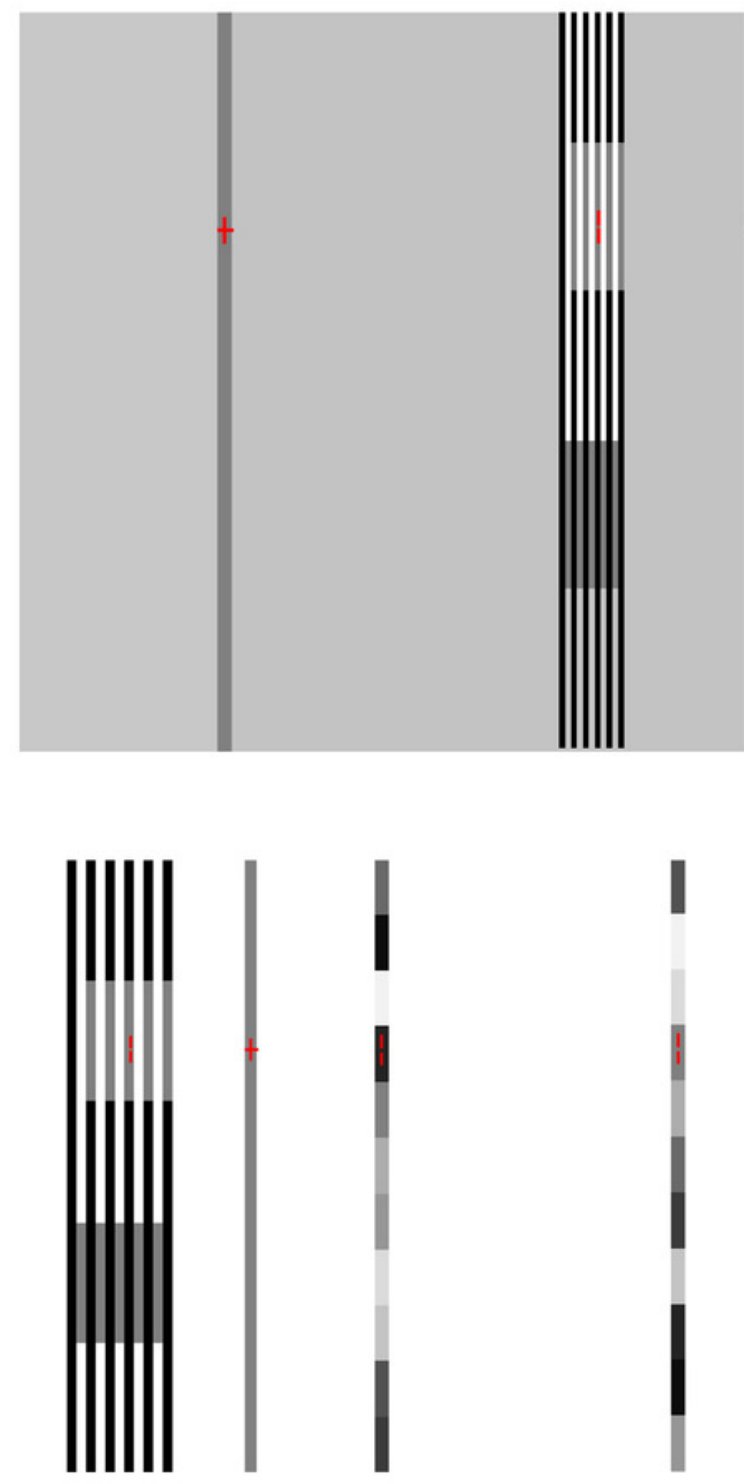

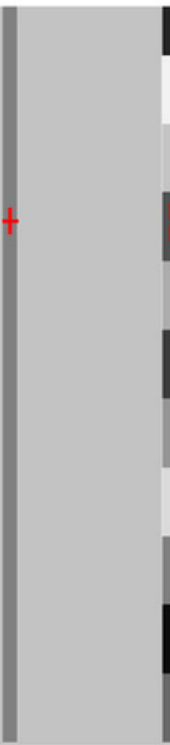

if comparator intensity > standard intensity then press 1 else press 2

(a)

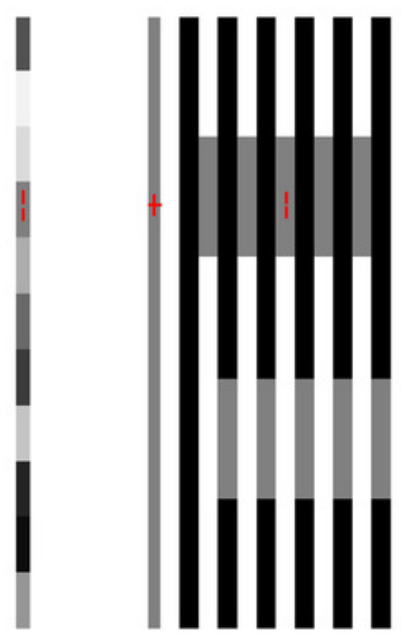

(b) 


\section{Figure 4}

Psychophysical experimental result: Average Psychometric functions for different spatial frequencies are displayed in different colors.

Figure 4. Psychophysical experimental result: Average Psychometric functions for the different spatial frequencies are displayed in different colors. For a particular spatial frequency, the upper curve represents the condition when the comparator appears brighter and the lower curve represents the condition when the comparator appears darker. While drawing the Psychometric function, a pair of curves is placed symmetrically against the luminance difference value of 0 . Figure 4 (a) gives the curves fitted with FitPsycheCurveLogit function. Figure 4(b) gives the curves fitted with modified function by Wichmann and Hill (2001). Figure 4(c) gives the average Psychometric function for six experimenters at a spatial frequency $2.97 \mathrm{cpd}$. The maximum and minimum deviation of perceived brightness for the individual experimenters are represented by the error bar at different luminance difference. Figure 4 (d) gives the Perceived enhancement in percentage of the points of subjective equality for different stimulus width. 


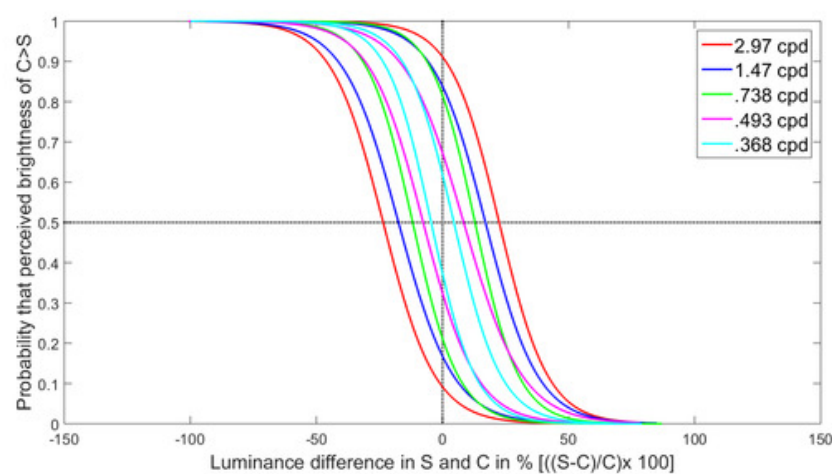

(a)

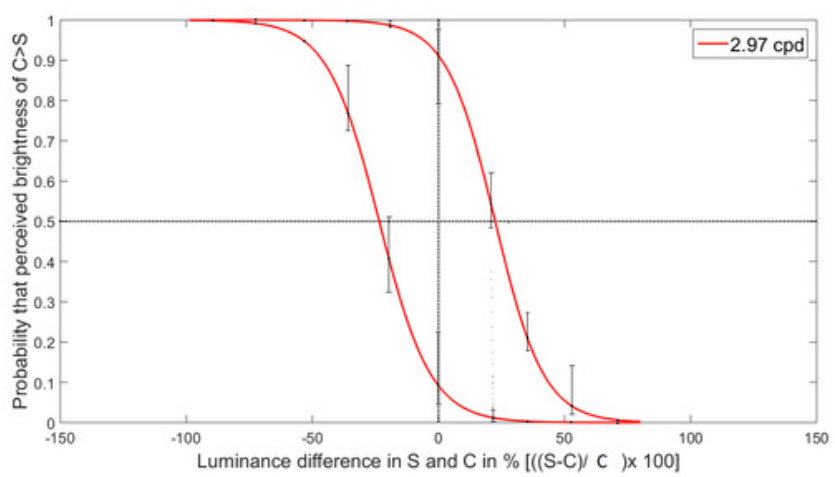

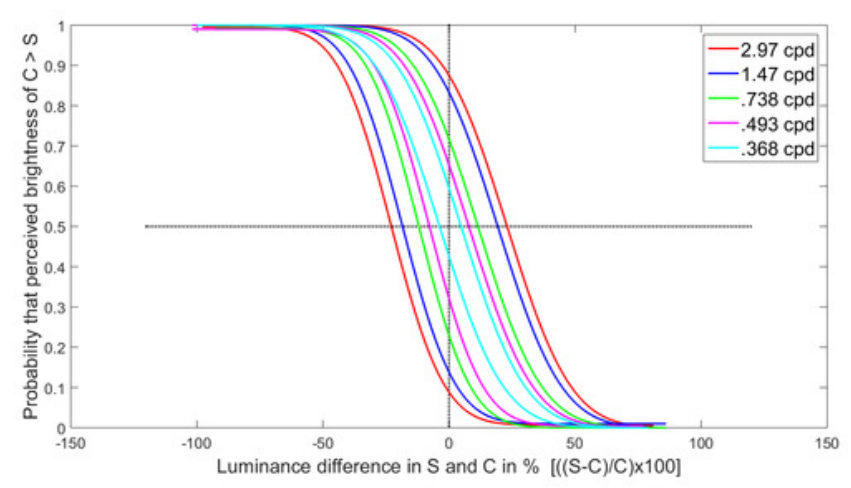

(b)

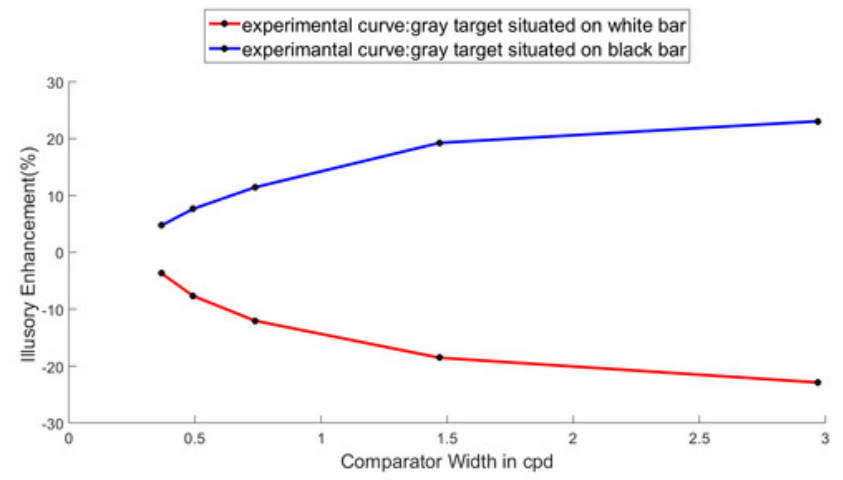




\section{Figure 5}

Psychophysical experimental result: Average Psychometric functions for the different length of the gray patches are displayed in different colors.

Figure 5: Psychophysical experimental result: Average Psychometric functions for the different length of the gray patches are displayed in different colors. While drawing the Psychometric function, a pair of curves is placed symmetrically against the luminance difference value of 0 . In Figure 5(a) the width of the comparator is is 8 pixels i.e. $0.738 \mathrm{cpd}$ and in Figure $5(\mathrm{~b})$ the width of the comparator is 4 pixels i.e. $1.47 \mathrm{cpd}$ 
Comparator width is 8 pixels i.e. $0.738 \mathrm{cpd}$

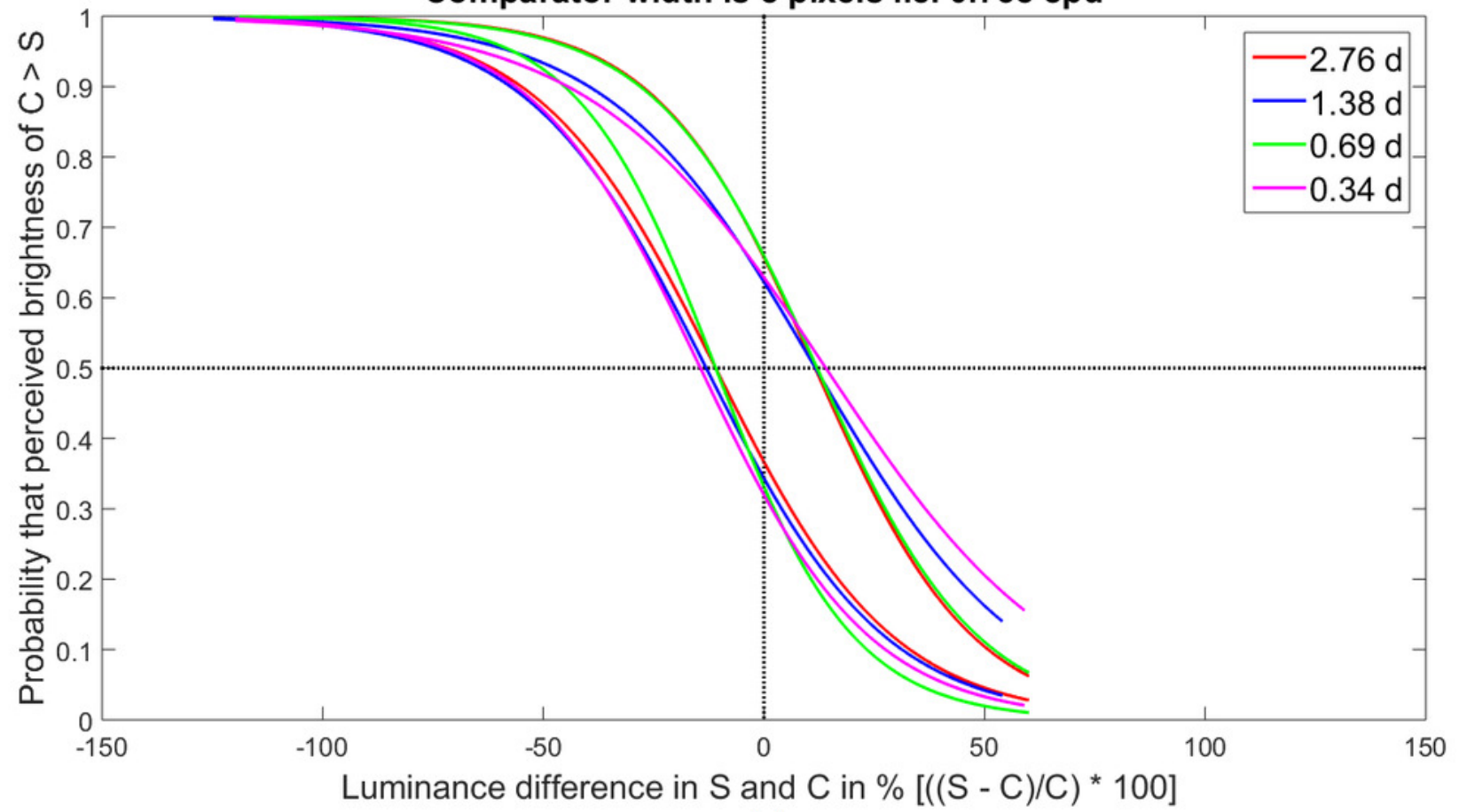

(a)

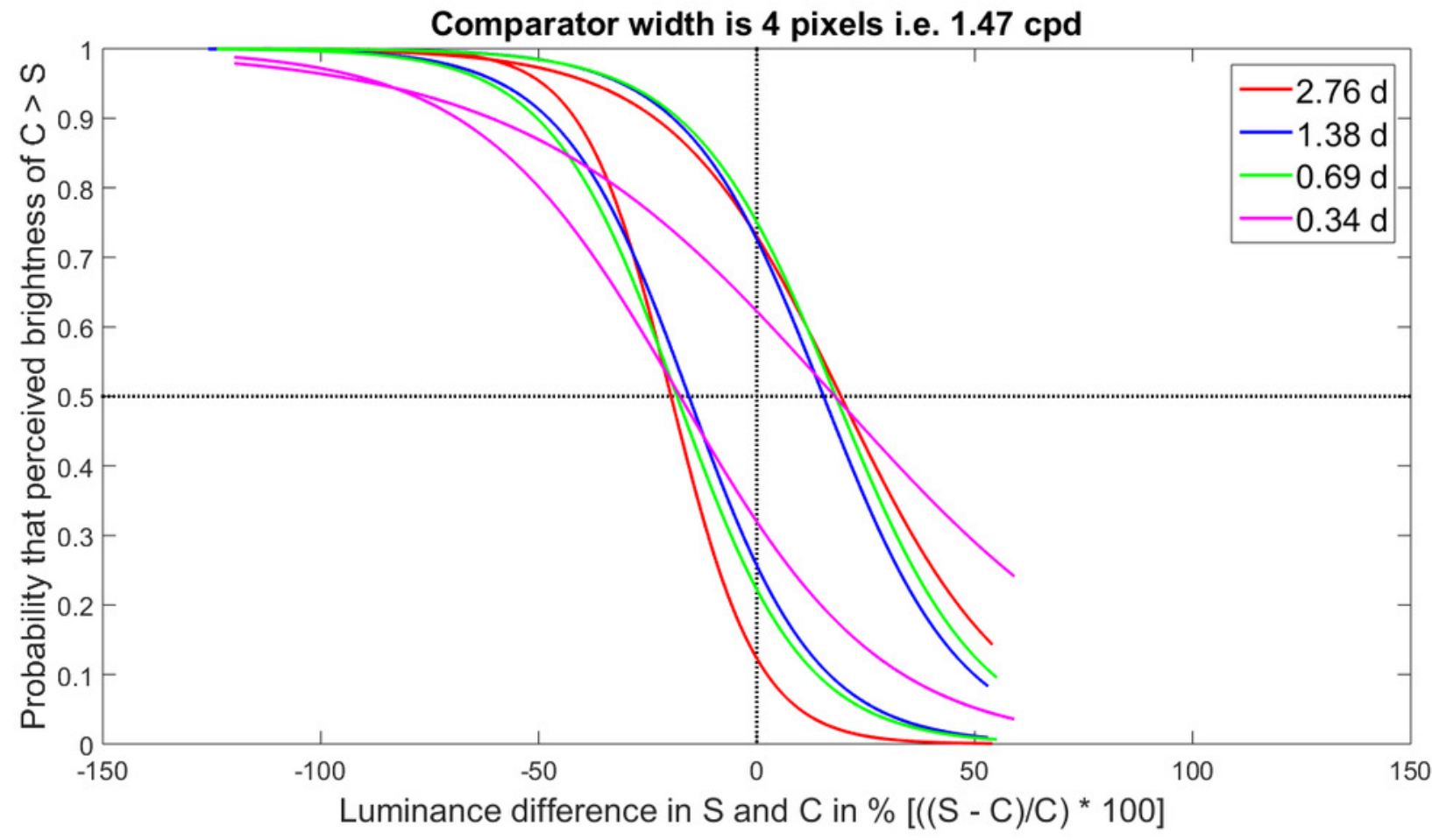

(b) 
Figure 6

Perceived enhancement for different target length.

Figure 6. Perceived enhancement in percentage of the points of subjective equality for different target length.

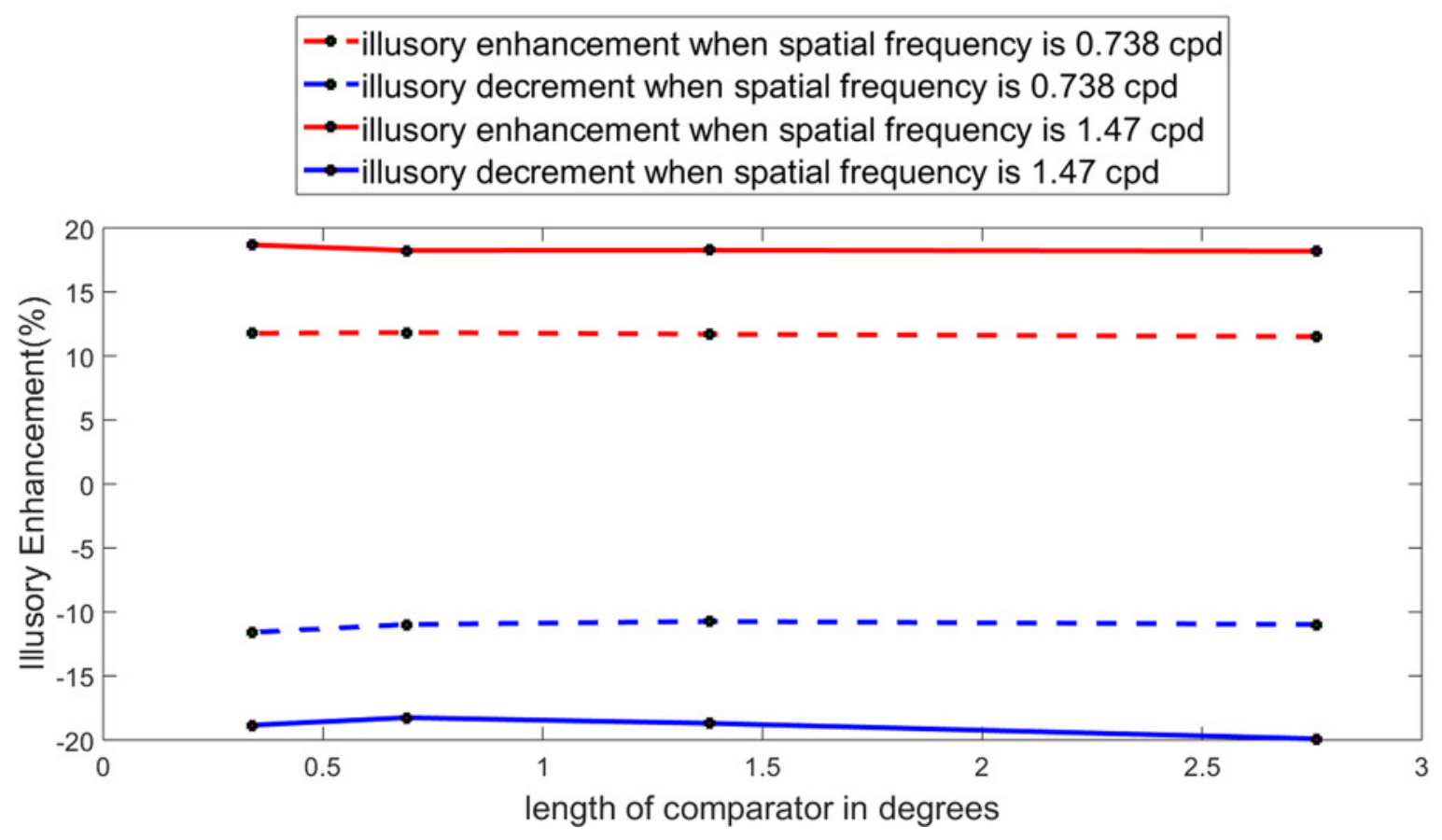




\section{Figure 7}

Experimental data on \% illusory enhancement as a function of length

Figure 7. Experimental data on \% illusory enhancement as a function of length of gray target in degrees has been plotted.

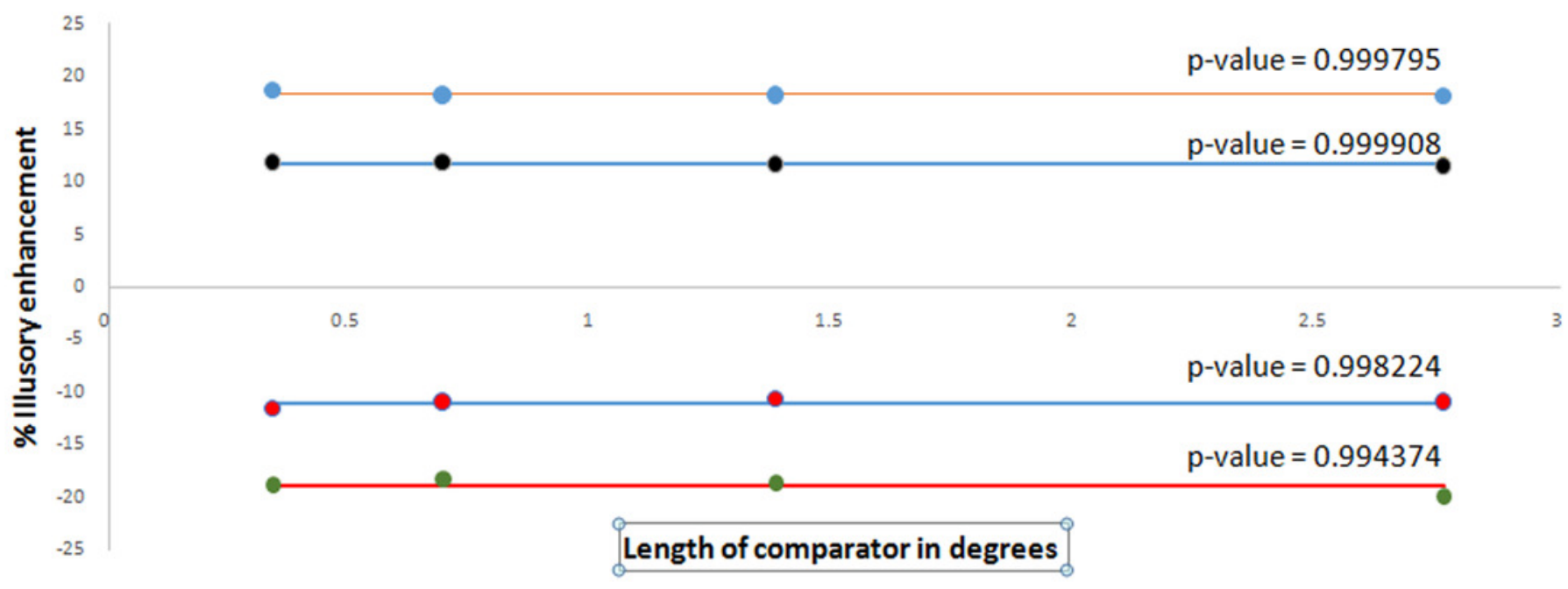


Figure 8

Variation of the illusory enhancement (\%) or the convolution response (\%) with spatial frequency of the inducing grating.

Figure 8. Variation of the illusory enhancement (\%) or the convolution response (\%) with spatial frequency of the inducing grating. The value of $\sigma$ is varied from 3.6 to 0.8 as the spatial frequency is increased. The simulated data is normalized against the intensity value of 128. The continuous curves represent the experimental results while the dotted curves are the outcome of the computer simulation.

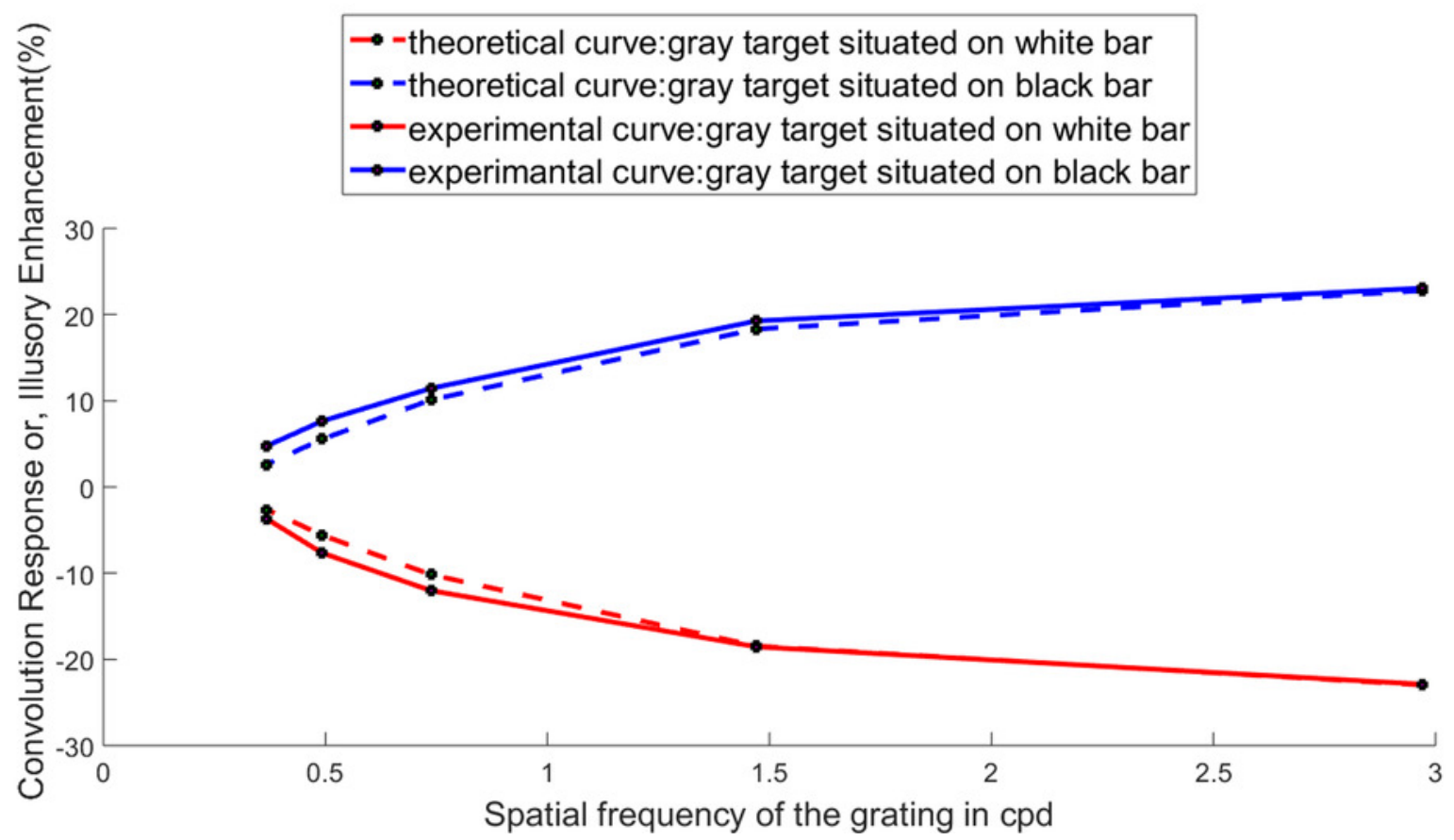


Figure 9

Percentage convolution response for different length of the target while keeping the spatial frequency as fixed.

Figure 9. Percentage convolution response for different length of the target while keeping the spatial frequency as fixed. In the graph, the red, blue, green, cyan and magenta curves show the simulated output at $2.97 \mathrm{cpd}, 1.47 \mathrm{cpd}, 0.738 \mathrm{cpd}, 0.493 \mathrm{cpd}$ and $0.368 \mathrm{cpd}$ respectively.

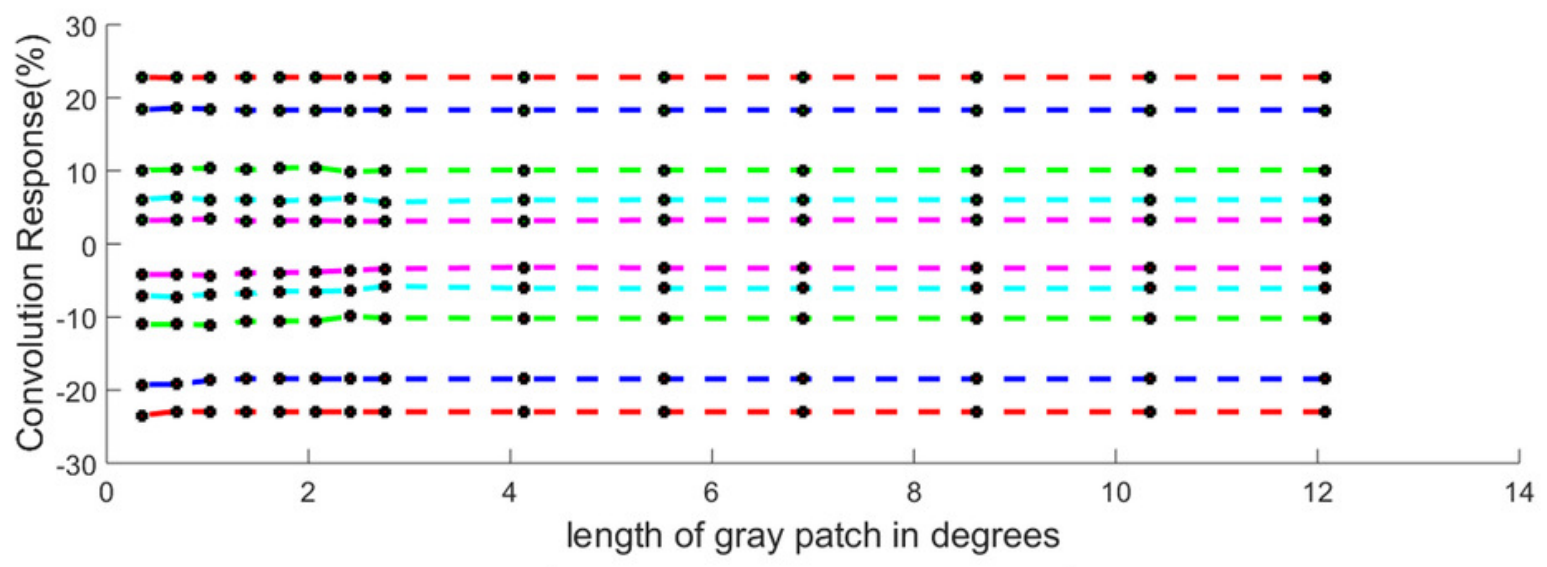

$\rightarrow$-spatial frequency $2.97 \mathrm{cpd}$ $\rightarrow$-spatial frequency $1.47 \mathrm{cpd}$ - -spatial frequency $0.738 \mathrm{cpd}$

- -spatial frequency $0.493 \mathrm{cpd}$

$\rightarrow$-spatial frequency $0.368 \mathrm{cpd}$ 


\section{Figure 10}

White's illusion at four different frequencies separated by a uniform gray background.

Figure 10. (a) White's illusion at four different frequencies separated from one another by a uniform gray background. 10 (b) and 10 (c) represent one dimensional intensity profile corresponding to the lines $A A^{\prime}$ and $B B^{\prime}$ respectively.

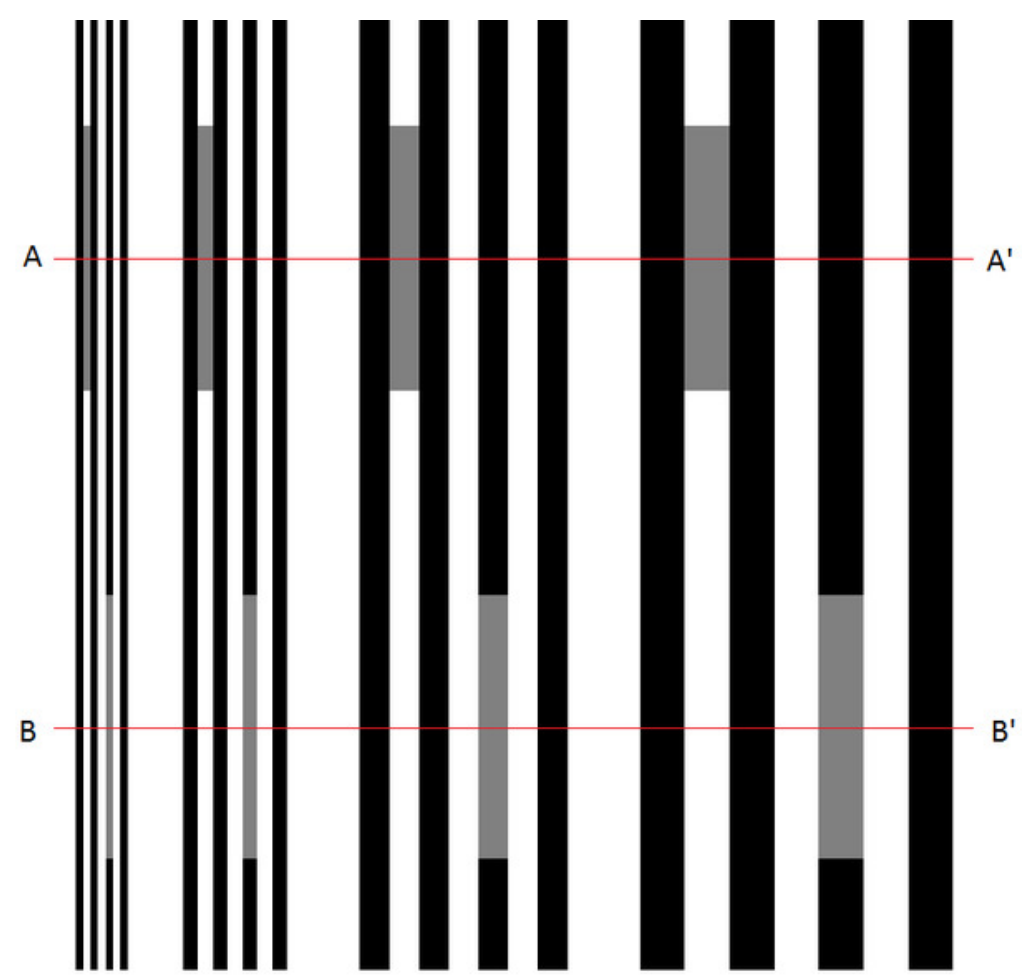

(a)

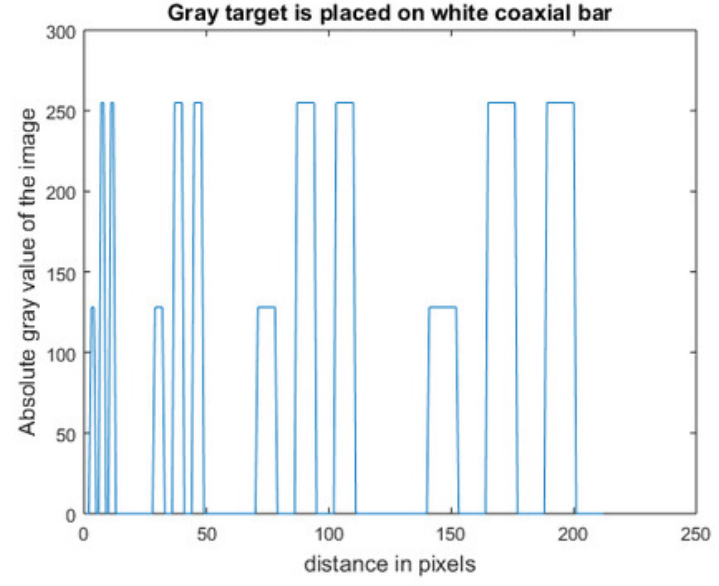

(b)

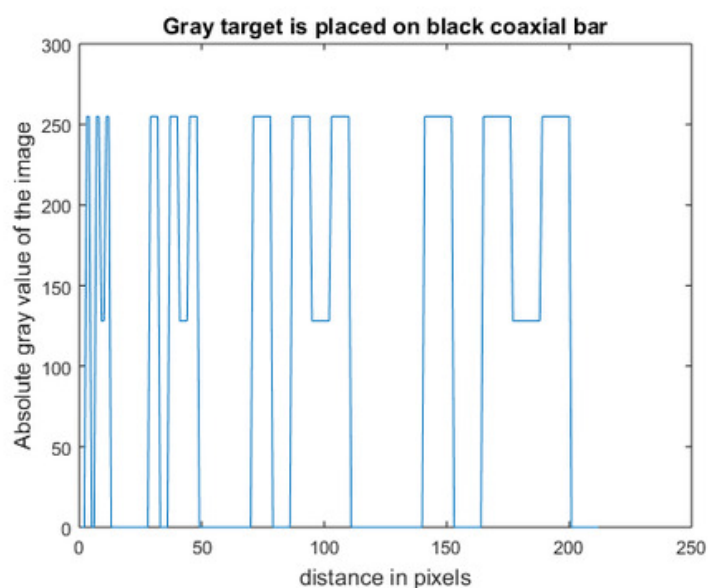

(c) 


\section{Figure 11}

The effect of Gaussian filtering with adaptive scale factors on figures 10(a), 10(b) and 10 (c).

Figure 11. shows the effect of Gaussian filtering with adaptive scale factors on figures 10(a), 10 (b) and 10 (c). The illusory effect at different frequencies can be differentiated by the naked eye. (a) gives convolved image of figure 10(a) with Gaussian filtering. Figures (b) and (c) shows the effect of Gaussian convolution on figures 10 (b) and 10 (c) respectively. 


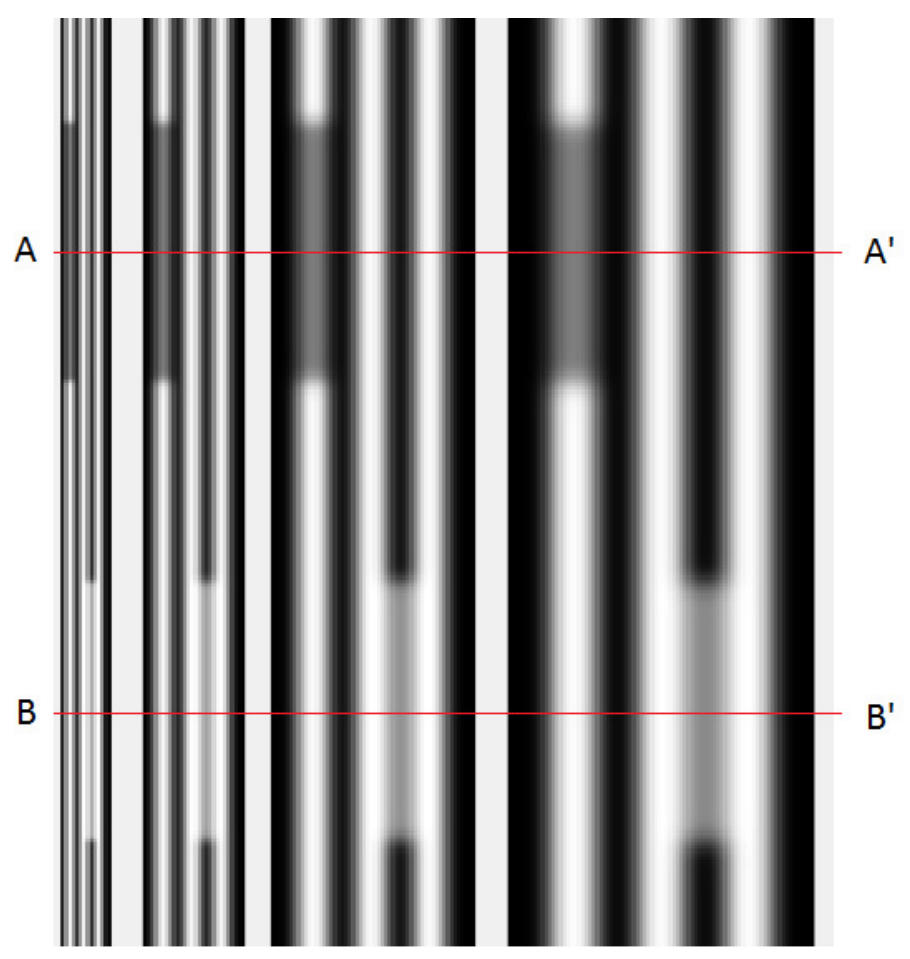

(a)

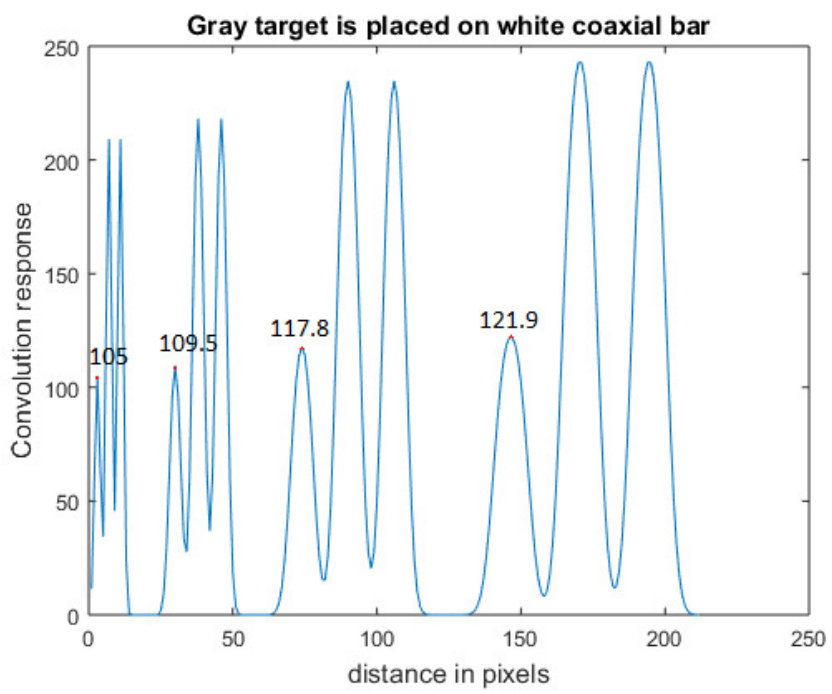

(b)

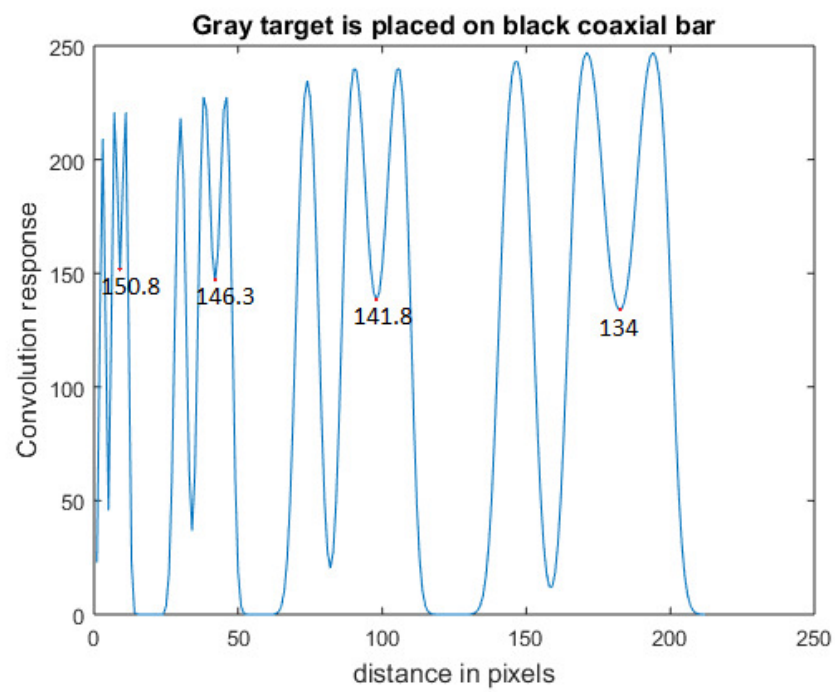

(c) 


\section{Table $\mathbf{1}$ (on next page)}

Parameters of the stimuli and the filter with varying spatial frequencies and the length of the gray patch

Table 1. Parameters of the stimuli and the filter used in the simulation with varying spatial frequencies and the length of the gray patch 


\begin{tabular}{|c|c|c|c|c|c|}
\hline \multirow{2}{*}{$\begin{array}{l}\text { Length of } \\
\text { gray patch } \\
\text { in pixels }\end{array}$} & \multicolumn{5}{|c|}{ Scale factor } \\
\hline & $\begin{array}{c}\text { Spatial } \\
\text { frequency } \\
=2.97 \mathrm{cpd}(2 \\
\text { pix }) \\
\text { Mask size }=(6 \\
\text { x6) }\end{array}$ & $\begin{array}{c}\text { Spatial } \\
\text { frequency } \\
=1.47 \mathrm{cpd}(4 \\
\text { pix }) \\
\text { Mask size } \\
=(12 \times 12)\end{array}$ & $\begin{array}{c}\text { Spatial } \\
\text { frequency } \\
=0.738 \mathrm{cpd}(8 \\
\text { pix }) \\
\text { Mask size } \\
=(20 \times 20)\end{array}$ & $\begin{array}{c}\text { Spatial } \\
\text { frequency } \\
=0.493 \\
\text { cpd(12 pix) } \\
\text { Mask size } \\
=(35 \times 35)\end{array}$ & $\begin{array}{c}\text { Spatial } \\
\text { frequency } \\
=0.368 \mathrm{cpd}(16 \\
\text { pix }) \\
\text { Mask size } \\
=(50 \times 50)\end{array}$ \\
\hline 70 & 0.8 & 1.4 & 2.3 & 3 & 3.6 \\
\hline 60 & 0.8 & 1.4 & 2.3 & 3 & 3.6 \\
\hline 50 & 0.8 & 1.4 & 2.3 & 3 & 3.6 \\
\hline 40 & 0.8 & 1.4 & 2.3 & 3 & 3.6 \\
\hline 32 & 0.8 & 1.4 & 2.3 & 3 & 3.6 \\
\hline 24 & 0.8 & 1.4 & 2.3 & 3 & 3.6 \\
\hline 16 & 0.8 & 1.4 & 2.3 & 3.1 & 5.7 \\
\hline 14 & 0.8 & 1.4 & 2.3 & 3.6 & 7.3 \\
\hline 12 & 0.8 & 1.4 & 2.4 & 4.9 & 8.5 \\
\hline 10 & 0.8 & 1.4 & 2.7 & 6.15 & 9.5 \\
\hline 8 & 0.8 & 1.41 & 3.8 & 7.2 & 10.4 \\
\hline 6 & 0.8 & 1.53 & 5.1 & 8.1 & 11.3 \\
\hline 4 & 0.81 & 2.35 & 6.35 & 9 & 12.1 \\
\hline 2 & 1.32 & 3.32 & 7.8 & 9.8 & 12.9 \\
\hline
\end{tabular}

